\title{
The single-peaked domain revisited: A simple global characterization
}

by Clemens Puppe

No. 97 | NOVEMBER 2016

\section{WORKING PAPER SERIES IN ECONOMICS}

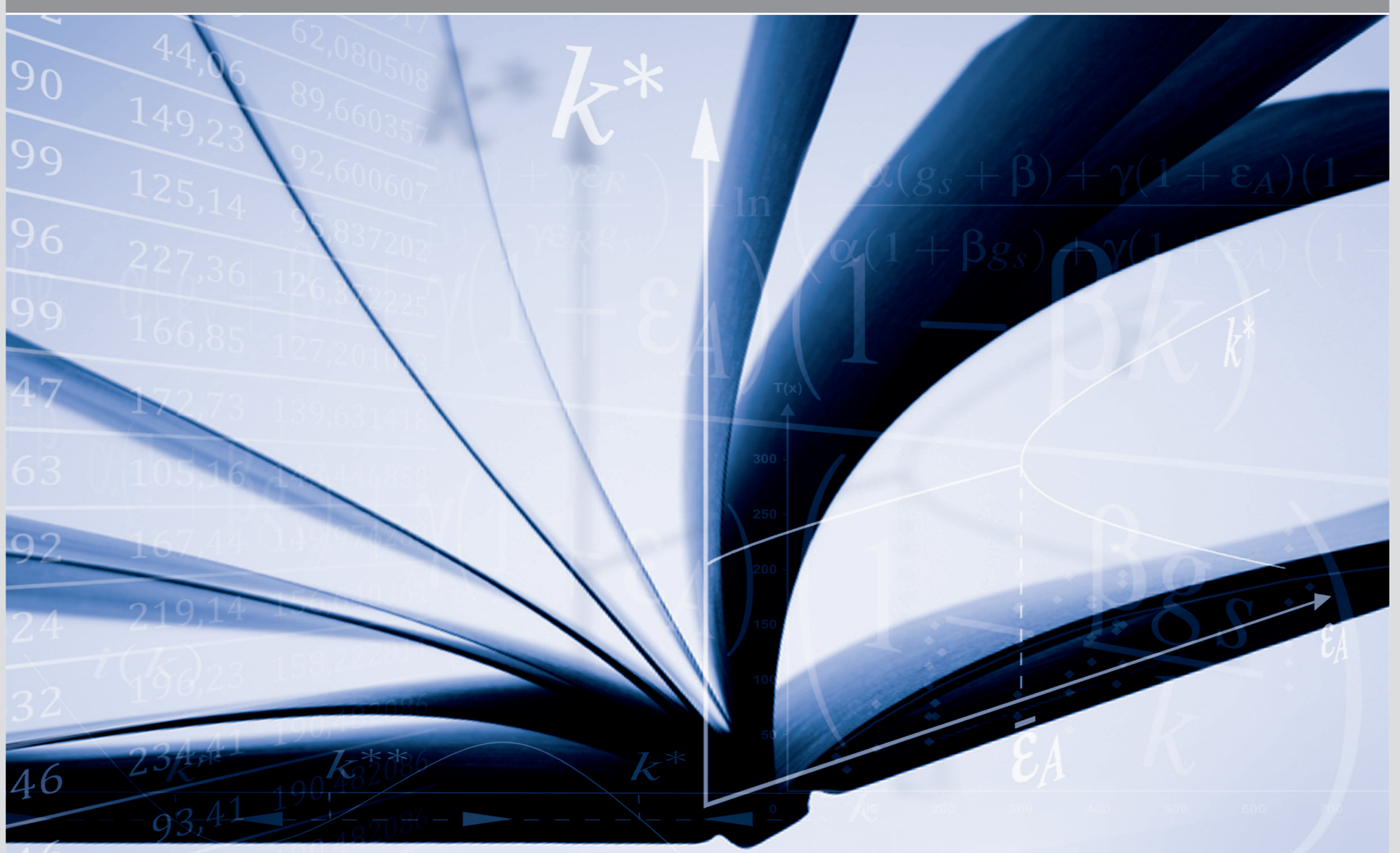




\section{Impressum}

Karlsruher Institut für Technologie (KIT)

Fakultät für Wirtschaftswissenschaften

Institut für Volkswirtschaftslehre (ECON)

Schlossbezirk 12

76131 Karlsruhe

KIT - Die Forschungsuniversität in der Helmholtz-Gemeinschaft

Working Paper Series in Economics

No. 97, November 2016

ISSN 2190-9806

econpapers.wiwi.kit.edu 


\title{
The Single-Peaked Domain Revisited: A Simple Global Characterization*
}

\author{
Clemens Puppe \\ Department of Economics and Management \\ Karlsruhe Institute of Technology (KIT) \\ D - 76131 Karlsruhe, Germany \\ clemens.puppe@kit.edu
}

October 2016

\footnotetext{
${ }^{*}$ I am indebted to Tobias Dittrich and Michael Müller who provided excellent research assistance. Tobias Dittrich also created the figures based on a graphic tool developed by David McCooey. This work was presented in seminars at Higher School of Economics in Moscow, Université de Cergy-Pontoise, Université Paris Dauphine, the University of Glasgow, at the Workshop on Game Theory and Social Choice at Corvinus University Budapest, at the "Tagung des Theoretischen Ausschusses des Vereins für Socialpolitik" in Basel and at the COMSOC conference in Toulouse. I am grateful to the participants for valuable discussion and comments. Special thanks to Ulle Endriss, Martin Hellwig, Anke Gerber, Gleb Koshevoy, Jérôme Lang, Jean-Francois Laslier, Bernard Monjardet, Hervé Moulin, Stefan Napel, Klaus Nehring, Georg Nöldeke, Matias Nunez, Hans Peters, Marcus Pivato, Anup Pramanik, Ernesto Savaglio, Attila Tasnádi and Bill Zwicker for particularly helpful comments.
} 
Abstract It is proved that, among all restricted preference domains that guarantee consistency (i.e. transitivity) of pairwise majority voting, the single-peaked domain is the only minimally rich and connected domain that contains two completely reversed strict preference orders. It is argued that this result explains the predominant role of single-peakedness as a domain restriction in models of political economy and elsewhere. The main result has a number of corollaries, among them a dual characterization of the single-dipped domain; it also implies that a single-crossing ('order-restricted') domain can be minimally rich only if it is a subdomain of a single-peaked domain. The conclusions are robust as the results apply both to domains of strict and of weak preference orders, respectively.

\section{JEL Classification D71, C72}

Keywords: Social choice, restricted domains, Condorcet domains, single-peakedness, single-dippedness, majority voting, single-crossing property. 


\section{Introduction}

A subset of preference orders on a finite set of alternatives is called single-peaked if there exists a left-to-right arrangement of alternatives such that all upper contour sets are connected ('convex') with respect to the given left-to-right arrangement of alternatives. The celebrated median voter theorem of Black [1948] and Arrow [1951] states that the domain of all single-peaked linear orders with respect to a fixed underlying spectrum of alternatives form a 'Condorcet domain,' i.e. pairwise majority voting with an odd number of individuals each of whom has preferences from the given domain induces a transitive relation. Moreover, the domain of all single-peaked preferences is minimally rich in the sense that every alternative is on top of at least one preference ordering; it is connected in the sense that every two single-peaked orders can be obtained from each other by a sequence of transpositions of neighboring alternatives such that the resulting order remains single-peaked at each step; and it contains two completely reversed orders (namely, the order that has the left-most alternative at the top and the order that has the right-most alternative at the top).

This paper's main result (Theorem 1) shows that, conversely, every minimally rich and connected Condorcet domain which contains at least one pair of completely reversed orders must be single-peaked. ${ }^{1}$ As is easily seen, any single-peaked domain contains at most one pair of completely reversed orders. We thus obtain as a corollary that, for any given pair of completely reversed orders, there is a unique maximal Condorcet domain that contains them and is minimally rich as well as connected: the domain of all orders that are single-peaked with respect to either one of the given pair of completely reversed orders (Corollary 1).

This result is remarkable in particular in view of the fact that quite a number of nonsingle-peaked Condorcet domains have been identified in the literature, among others the domains satisfying Sen's 'value restriction' condition (Sen [1966]) with the 'single-dipped' domain (Inada [1964]) as a special case, the domains satisfying the so-called 'intermediateness' property (Grandmont [1978], Demange [2012]), and the 'order-restricted' domains identified by Rothstein [1990]; the latter domains are sometimes also referred to as the domains with the single-crossing property (Gans and Smart [1996], Saporiti [2009], Puppe and Slinko [2015]). Our analysis shows that none of these domains can jointly satisfy the three conditions of minimal richness, connectedness and the inclusion of a pair of completely reversed orders unless it is also single-peaked. In particular, a single-crossing domain can be minimally rich only if it is at the same time single-peaked (Corollary 3).

The purpose of the present analysis is not to justify the assumption of single-peakedness per se and, in fact, the empirical evidence for single-peakedness is mixed, see the review of the literature below. The main argument put forward here is that, among all domains that guarantee consistency of pairwise majority voting, the single-peaked domain is distinguished by a remarkably simple set of additional requirements: connectedness, minimal richness and the existence of two completely reversed orders. The main conclusion to be

\footnotetext{
${ }^{1}$ In fact, as detailed in Section 2 below, the condition of connectedness can be substantially relaxed in this result to the condition that there exist one path that connects a pair of completely reversed orders.
} 
drawn from the present analysis is therefore that, if a modeler wishes to guarantee transitivity of the majority relation for any possible profile of agents' preferences, then the assumption of single-peakedness follows very naturally. In this sense, the present study may thus be interpreted as a conditional defense of single-peakedness. ${ }^{2}$

The results presented here are robust as they generalize with some additional work, but using the same underlying logic, to the case of weak preference orders, i.e. to the case in which individual preferences may display indifferences. In this case, the domain of all (weakly) single-peaked weak orders does not form a Condorcet domain in our sense since, even with an odd number of voters, the indifference relation corresponding to pairwise majority voting may be intransitive. Moreover, the notion of connectedness has to be suitably adapted since two 'neighboring' weak orders may differ in the ranking of more than one pair of alternatives if one of these orders displays an indifference class with more than two elements. Nevertheless, we still obtain that any connected, minimally rich Condorcet domain that contains (at least) two completely reversed strict orders must be single-peaked with respect to either one of the pair of completely reversed orders (Theorem 3). However, if one allows for indifferences - in particular also on the top of individual preferences - there are different possibilities to formulate the condition of minimal richness. If one only requires that each alternative be among the top elements of at least one order in the domain, the stated conditions characterize a class of different non-isomorphic maximal Condorcet domains, all of which are proper subdomains of the domain of all single-peaked orders. If, on the other hand, one imposes the stronger requirement that each alternative be the unique top element of at least one order in the domain, the stated conditions characterize a unique maximal Condorcet domain of singlepeaked orders (with respect to the left-to-right arrangement of alternatives given by either one of the completely reversed strict orders).

The above characterization results of the single-peaked domain imply 'dual' characterization results for the single-dipped domain in a straightforward way, both in the case of linear and and in the case of weak orders. Specifically, any connected Condorcet domain containing two completely reversed orders such that every alternative is (among) the least preferred alternative(s) for some order in the domain must be single-dipped (see Theorems 2 and 4).

\section{Relation to the literature}

The literature on single-peaked preferences is abundant both in economics and political science. Their application ranges from the Hotelling-Downs model of political competition to models of local public good provision (for a modern treatment see, e.g., Austen-Smith and Banks [1998]). It is well-known that the assumption of single-peakedness enables possibility results both in the theory of preference aggregation (Black [1948], Arrow [1951]) and in the theory of strategy-proof social choice (Moulin [1980]). Moreover, it has frequently been argued that the assumption of single-peakedness is reasonable in contexts

\footnotetext{
${ }^{2}$ Of course, by contraposition, the same argument transforms potential doubts about the validity of single-peakedness in specific contexts into corresponding doubts on the existence of consistent pairwise majorities at all in these contexts.
} 
in which alternatives are naturally arranged according to an exogenous one-dimensional scale, e.g., in terms of political views on a left-to-right spectrum, or in terms objective distance, temperature, etc. The empirical evidence on single-peakedness is mixed. Some authors have argued that a tendency towards single-peakedness may be assumed under certain circumstances, in particular when there is repeated interaction and/or 'deliberation' (see, e.g., Spector [2000], DeMarzo et al. [2003], List et al. [2013]). Others have cast doubt on the applicability of single-peakedness, in particular in cases where compromises are difficult to reach and a search for them threatens to lead to a deadlock (Egan [2014]).

The paper in the literature that is closest to the present analysis is Ballester and Haeringer [2011]. These authors also provide an axiomatic characterization of the singlepeaked domain (though only in the case of linear orders). ${ }^{3}$ However, the conditions employed by these authors are very different in character from the ones used here. Specifically, Ballester and Haeringer [2011] use two families of conditions. The first is the condition that among every triple of alternatives their should be at least one that is never the worst of the three for any voter. This is one of Sen's family of 'value restrictions' (Sen [1966]), and it evidently amounts to assuming single-peakedness on all triples, hence it directly implies transitivity of the majority relation. However, it is also known that single-peakedness on all triples ('local single-peakedness') is not sufficient to guarantee single-peakedness globally (cf. Inada [1964]). Therefore, additional conditions are needed to characterize the single-peaked domain. The important contribution of Ballester and Haeringer [2011] is to show that the absence of a certain preference constellation on all quadruples of alternatives does the job. By contrast, the present analysis only assumes transitivity of the majority relation corresponding to every profile with an odd number of voters, and derives the single-peakedness of the domain from the three conditions of connectedness, minimal richness and the existence of two completely reversed orders (either of which then represents the underlying left-to-right spectrum). The latter three conditions are global properties of a domain, hence the title of this paper. By contrast, all conditions used by Ballester and Haeringer [2011] are 'local' conditions as they apply simultaneously either to all triples or to all quadruples of alternatives. While the conditions of the present analysis can be suitably adapted to yield a corresponding characterization of the weakly single-peaked domain, it is not obvious how to appropriately formulate Ballester's and Haeringer's local conditions in the case of weak orders.

The present study also informs the literature that aims at identifying 'large' Condorcet domains, see the excellent survey Monjardet [2009] and the more recent work on this topic by Danilov et al. [2012], Danilov and Koshevoy [2013]. Indeed, our main result suggests that Condorcet domains with the maximal number of elements on a given set of alternatives, the so-called maximum Condorcet domains, are most likely not minimally rich. This may seem particularly surprising, as it is known that that the cardinality of a maximum Condorcet domain exceeds the cardinality of the domain of all single-peaked domain considerably; for instance, with $n$ alternatives a single-peaked domain has at most $2^{n-1}$ elements, while the cardinalities of the maximum Condorcet domains are 9, 20, 45,

\footnotetext{
${ }^{3}$ Strictly speaking, Ballester and Haeringer [2011] characterize the domain of all single-peaked profiles which is a slightly different task.
} 
for $n=4,5,6$, respectively, and these numbers are all attained by connected Condorcet domains containing a pair of completely reversed orders. The general structure and precise cardinality of the maximum Condorcet domains for larger $n$ is unknown. However, it is known that the largest cardinality of a connected Condorcet domain on $n$ alternatives that contains two completely reversed orders always exceeds $2^{n-1}$, see Fishburn [1997]. By this paper's main result, the corresponding domains can never be minimally rich.

We finally emphasize the implications of our analysis for the literature on the singlecrossing property. In the present finite framework, a domain is said to have the singlecrossing property if the agents can be arranged in a fixed linear order such that, for every pair of alternatives, if two voters prefer one alternative to the other, then so do all agents that are between them in the given linear order of voters. ${ }^{4}$ It has frequently been noted that single-peakedness and the single-crossing property in this sense are logically independent conditions, see, e.g., Saporiti [2009]. However, since every single-crossing domain can be extended to a connected single-crossing domain containing two completely reversed orders, our main result yields as a corollary that all minimally rich single-crossing domains must also be single-peaked, i.e. a subset of the domain of all single-peaked orders (cf. Elkind et al. [2014]). Since for $n>3$, only proper subsets of the domain of all single-peaked preferences can have the single-crossing property, this has the further interesting consequence that no minimally rich single-crossing domain can constitute a maximal Condorcet domain.

The remainder of this paper is organized as follows. In Section 2 we state and prove our main result in the case of linear ('strict') orders. We also show by means of examples that none of the conditions can be omitted in the characterization of the single-peaked domain. Section 3 generalizes the characterization to the case of weak orders. In the appendix, it is shown that, for all 'closed' (in particular, maximal) Condorcet domains containing at least one pair of completely reversed orders, the condition of connectedness is implied by the substantially weaker property that there be at least one path connecting a pair of completely reversed orders; and that this weaker property in fact not only implies connectedness but the stronger property of 'direct' connectedness. (This holds both in the case of linear and weak orders.)

\section{Characterizing the single-peaked domain: The case of strict preference orders}

\subsection{Statement of main result}

Consider a finite set of alternatives $X$ and the set $\mathcal{P}(X)$ of all linear (strict) orders (i.e., complete, transitive and antisymmetric binary relations) on $X$. A subset $\mathcal{D} \subseteq \mathcal{P}(X)$ will be called a domain of preferences or simply a domain. A profile $\pi=\left(P_{1}, \ldots, P_{n}\right)$ on $\mathcal{D}$ is an

\footnotetext{
${ }^{4}$ This condition is related to but prima facie different from the well-known Spence-Mirrlees 'singlecrossing' condition which requires that agents' types are unambiguously ordered according to their marginal rate of substitution uniformly across the good space.
} 
element of the Cartesian product $\mathcal{D}^{n}$ for some number $n \in \mathbb{N}$ of 'voters,' where the linear order $P_{i}$ represents the preferences of the $i$ th voter over the alternatives from $X$. A profile with an odd number of voters will simply be referred to as an odd profile. Frequently, we will denote linear orders simply by listing the alternatives in descending order, e.g. the linear order that ranks $a$ first, $b$ second, $c$ third, etc., is denoted by $a b c \ldots$.

The majority relation associated with a profile $\pi$ is the binary relation $P_{\pi}^{\text {maj }}$ on $X$ such that $x P_{\pi}^{\mathrm{maj}} y$ if and only if more than half of the voters rank $x$ above $y$. Note that, according to this definition, the majority relation is asymmetric and for any odd profile $\pi$ and any two distinct alternatives $x, y \in X$, we have either $x P_{\pi}^{\mathrm{maj}} y$ or $x P_{\pi}^{\mathrm{maj}} y$. The class of domains $\mathcal{D} \subseteq \mathcal{P}(X)$ such that, for all odd $n$, the majority relation associated with any profile $\pi \in \mathcal{D}^{n}$ is transitive has received significant attention in the literature, see the excellent survey of Monjardet [2009] and the references therein. In the following, we will refer to any such domain as a Condorcet domain. A domain $\mathcal{D}$ is called a maximal Condorcet domain if every Condorcet domain (on the same set of alternatives) that contains $\mathcal{D}$ as a subset must in fact coincide with $\mathcal{D}$. It is well-known that any maximal Condorcet domain $\mathcal{D}$ is closed in the sense that the majority relation of any odd profile from $\mathcal{D}$ is again an element of $\mathcal{D}$ (and not only of $\mathcal{P}(X)$ ), cf. [Puppe and Slinko, 2015, Lemma 2.1].

A domain $\mathcal{D}$ is single-peaked with respect to the linear order $>$ on $X$ if, for all $P \in \mathcal{D}$ and all $w \in X$, the upper contour sets $U_{P}(w):=\{y \in X: y P w\}$ are connected ('convex') in the order $>$, i.e. $\{x, z\} \subseteq U_{P}(w)$ and $x<y<z$ jointly imply $y \in U_{P}(w)$. A domain $\mathcal{D}$ is simply called single-peaked if there exists some linear order $>$ such that $\mathcal{D}$ is single-peaked with respect to $>$. The domain of all orders that are single-peaked with respect to the fixed order $>$ on $X$ is denoted by $\mathcal{S P}_{>}(X)$. If a domain is single-peaked with respect to $>$, we will often call the linear order $>$ the spectrum underlying the single-peaked domain.

A path in $\mathcal{P}(X)$ is subset $\left\{P_{1}, \ldots, P_{m}\right\} \subseteq \mathcal{P}(X)$ with $m \geq 2$ such that for all $j=$ $1, \ldots, m-1$, the two consecutive orders $P_{j}$ and $P_{j+1}$ differ in the ranking of exactly one pair $x, y$ of (distinct) alternatives; note in that case $x$ and $y$ must be adjacent alternatives in both orders $P_{j}$ and $P_{j+1}$. A pair of orders which differ in the ranking of exactly one (adjacent) pair of alternatives will be called neighbors. A domain $\mathcal{D}$ will be called connected if, for every pair $P, P^{\prime} \in \mathcal{D}$ of distinct orders in $\mathcal{D}$, there exists a path $\left\{P_{1}, \ldots, P_{m}\right\}$ that connects $P$ and $P^{\prime}$ (i.e. $P_{1}=P$ and $P_{m}=P^{\prime}$ ) and that lies entirely in $\mathcal{D}$ (i.e. $P_{j} \in \mathcal{D}$ for all $j=1, \ldots, m)$.

Two orders $P$ and $P^{\text {inv }}$ are called completely reversed if $P$ and $P^{\text {inv }}$ rank the alternatives in $X$ in exactly the opposite way, i.e. for all distinct $x$ and $y, x P y \Leftrightarrow \operatorname{not}\left(x P^{\text {inv }} y\right)$. Note that by the completeness assumption, two orders $P, P^{\text {inv }} \in \mathcal{P}(X)$ are completely reversed if and only if $x P y \Leftrightarrow y P^{\mathrm{inv}} x$. A domain is said to have maximal width if it contains at least one pair of completely reversed orders. ${ }^{5}$ The following property may look artificial at first, but turns out to be conceptually very natural. Say that a domain $\mathcal{D} \subseteq \mathcal{P}(X)$ is semi-connected if it contains two completely reversed orders $P$ and $P^{\text {inv }}$ and an entire path connecting them (cf. [Danilov et al., 2012, p.938]). Evidently, semi-connectedness implies maximal width, and is implied by, but logically weaker than, the conjunction of

\footnotetext{
${ }^{5}$ Domains with that property are called 'normal' in Danilov and Koshevoy [2013].
} 
connectedness and maximal width. ${ }^{6}$ Finally, a domain $\mathcal{D}$ will be called minimally rich if, for every alternative $x \in X$, there exists an order $P \in \mathcal{D}$ such that $P$ has $x$ as the top alternative.

The following characterization of the single-peaked domain is this paper's main result.

Theorem 1. a) For every linear order $>$ on $X$, the domain $\mathcal{S P}_{>}(X)$ of all singlepeaked orders with respect to $>$ is a connected and minimally rich Condorcet domain with maximal width. (In particular, $\mathcal{S P}_{>}(X)$ is semi-connected.)

b) Conversely, let $\mathcal{D} \subseteq \mathcal{P}(X)$ be a semi-connected and minimally rich Condorcet domain. Then, $\mathcal{D}$ is single-peaked.

Except perhaps for the connectedness, the properties of the domain of all singlepeaked orders stated in part a) are straightforward to verify. Clearly, the two completely reversed orders are $>$ itself and its reverse. Note that a single-peaked domain can contain at most one pair of completely reversed orders, ${ }^{7}$ therefore such pair uniquely determines a corresponding maximal single-peaked domain, and we have the following corollary.

Corollary 1. Let $\mathcal{D} \subseteq \mathcal{P}(X)$ be a maximal Condorcet domain that is (semi-)connected, minimally rich and contains the pair $P, P^{\text {inv }}$ of completely reversed orders, then $\mathcal{D}=$ $\mathcal{S} \mathcal{P}_{>}(X)$ where the spectrum $>$ is given by either $P$ or $P^{\text {inv }}$.

Figure 1 below depicts the (unique) maximal single-peaked domain containing the pair $a b c d$ and $d c b a$ of completely reversed orders on the set $X=\{a, b, c, d\}$. Neighboring orders are connected by an edge; the domain consists of the orders marked in red color.

The proof of Theorem 1 is provided in Section 2.3 below. Note that in the sufficiency part b) it is not asserted that $\mathcal{D}$ must contain all orders that are single-peaked with respect to some given linear order (and this does in fact not follow); on the other hand, due to the semi-connectedness, any domain satisfying the conditions of Theorem 1b) must contain at least $\# X \cdot(\# X-1) / 2+1$ elements (because every ordered pair of alternatives has to be switched at least once on any path connecting two completely reversed orders).

Before providing the proof, we discuss the meaning and significance of the characterizing conditions in Theorem 1 . We will also show by means of concrete examples that the characterization of the single-peaked domain provided by Theorem 1 is tight in the sense that each condition in part b) is indeed necessary to obtain the conclusion.

\subsection{Discussion}

The conditions imposed on preference domains in Theorem 1 will now be discussed. In particular, we demonstrate by means of examples that each condition in part b) is necessary to obtain the single-peakedness of the domain. A secondary purpose of this subsection is to illustrate the great diversity of the class of (maximal) Condorcet domains.

\footnotetext{
${ }^{6}$ In fact, in the appendix it is shown that for 'closed' Condorcet domains semi-connectedness implies connectedness and is thus equivalent to the conjunction of connectedness and maximal width. This result is important for understanding the structure of Condorcet domains, but it is not needed for our main results and therefore deferred to the appendix (see also the remark in Subsection 2.3 below).

${ }^{7}$ This follows, e.g., immediately from Fact 2.2 below.
} 


\subsubsection{Consistency of majority voting}

The condition of consistency of pairwise majority voting lies of at the heart of the present analysis and 'defines' the problem. Transitivity of the majority relation (for odd profiles) is certainly a strong requirement but, as already noted in the introduction, the goal here is not to justify it but to study its implications. Clearly, the condition that $\mathcal{D}$ be a Condorcet domain is necessary for the conclusion that $\mathcal{D}$ is single-peaked. For instance, the universal domain $\mathcal{P}(X)$ evidently satisfies all other conditions in Theorem 1b), but the universal domain is clearly not single-peaked.

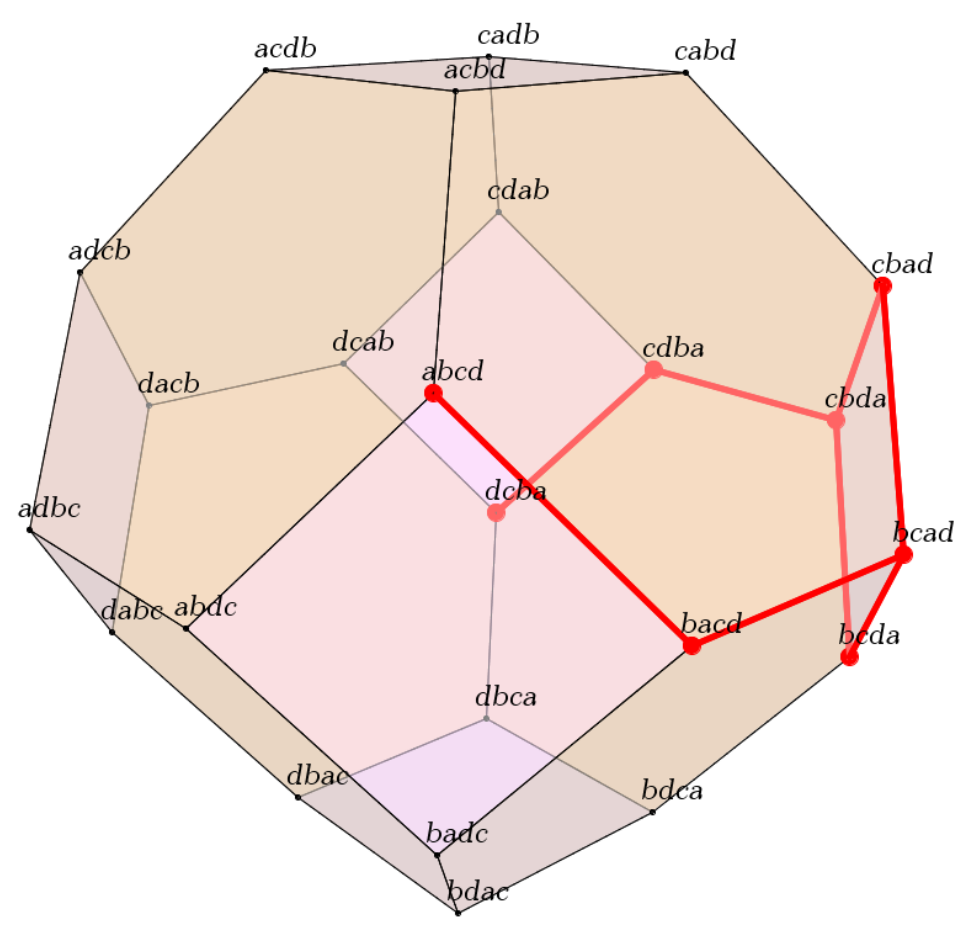

Fig. 1: A maximal single-peaked domain on $X=\{a, b, c, d\}$.

\subsubsection{Maximal width: The existence of two completely reversed orders}

One may think of a domain as the description of a 'society.' Under this interpretation, the existence of two orders in the domain that are completely reverses of each other is a condition of maximal 'width' of opinions. The underlying society is required to admit the most extreme opinions with respect to at least one dimension. As with the other conditions in Theorem 1, the maximal width condition describes a substantial requirement. ${ }^{8}$ On the other hand, it is not evident whether there are natural classes of maximal Condorcet domains that violate the maximal width condition. Figure 2 illustrates the necessity of the

\footnotetext{
${ }^{8}$ Also mathematically, it has significant consequences. Indeed, it is well-known that, together with the consistency of majority voting, maximal width implies that the domain can be embedded in a distributive lattice (cf. Abello [1991], Chameni-Nembua [1989], Monjardet [2009], Danilov and Koshevoy [2013], Puppe and Slinko [2015]).
} 
maximal width condition in Theorem 1b) by displaying a maximal Condorcet subdomain of $\mathcal{P}(X)$ on $X=\{a, b, c, d\}$ that is not single-peaked but connected as well as minimally rich. The connectedness and minimal richness of the depicted domain (the red marked orders) is evident. To verify that it is not single-peaked, note first that $a$ and $d$ are the only two alternatives that occur at the bottom of each marked order. Since abcd is a member, this implies that if the domain is to be single-peaked with respect to $>$, we must have either $a>b>c>d$ or $d>c>b>a$. However, in either case the contained order $b d c a$, for instance, would not qualify as single-peaked.

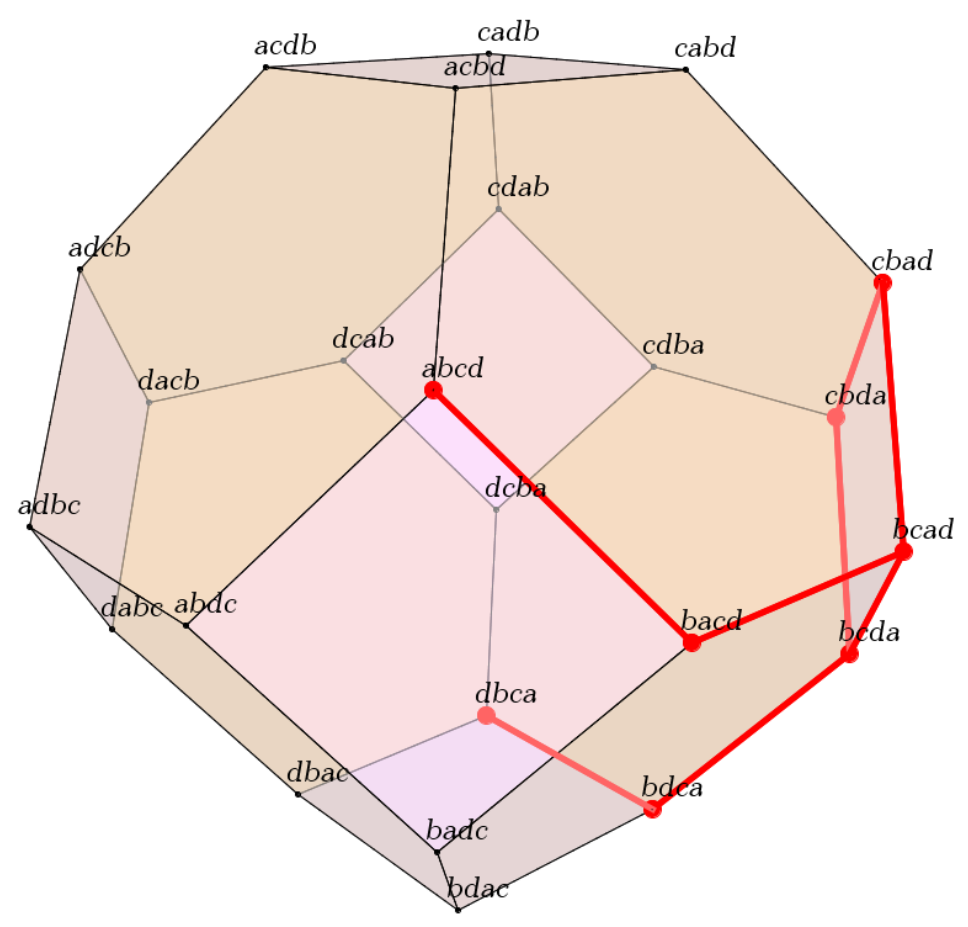

Fig. 2: A connected and minimally rich Condorcet domain without a pair of two completely reversed orders.

The fact that the domain depicted in Fig. 2 is indeed a Condorcet domain can be easily inferred from the following well-known result. ${ }^{9}$

Fact 2.1. Let $\mathcal{D} \subseteq \mathcal{P}(X)$ with $X$ finite. The following statements are equivalent.

i) $\mathcal{D}$ is a Condorcet domain, i.e. the majority relation corresponding to every odd profile on $\mathcal{D}$ is an element of $\mathcal{P}(X)$.

ii) The majority relation corresponding to every profile on $\mathcal{D}$ is acyclic. ${ }^{10}$

\footnotetext{
${ }^{9}$ See, e.g. [Monjardet, 2009, p. 142]). Condition iii) is Sen's [1966] 'value restriction' and condition iv) has been introduced by Ward [1965] as the 'absence of a Latin square' (in other terminology, it requires the absence of a 'Condorcet cycle'; cf. Condorcet, 1785). In light of this condition, Condorcet domains of (linear) orders are sometimes referred to as 'acyclic sets of linear orders' (e.g. by Fishburn [1997]).

${ }^{10} \mathrm{An}$ asymmetric binary relation $P$ is acyclic if there does not exist a subset $\left\{x_{1}, \ldots, x_{m}\right\} \subseteq X$ such that $x_{1} P x_{2}, x_{2} P x_{3}, \ldots, x_{m-1} P x_{m}$ and $x_{m} P x_{1}$.
} 
iii) In any triple $x, y, z \in X$ of pairwise distinct alternatives, there exists one element that either never has rank 1, or never has rank 2, or never has rank 3 in the restrictions of the orders in $\mathcal{D}$ to the set $\{x, y, z\}$.

iv) For no triple $P_{1}, P_{2}, P_{3} \in \mathcal{D}$, and no triple $x, y, z \in X$ of pairwise distinct alternatives one has $x P_{1} y P_{1} z, y P_{2} z P_{2} x$ and $z P_{3} x P_{3} y$.

We finally note that the domain depicted in Fig. 2 is in fact a maximal Condorcet domain. ${ }^{11}$

\subsection{3 (Semi-)Connectedness}

Continuing with the metaphor of a domain representing a society, connectedness has a clear meaning as well: it must be possible to reach any admissible opinion from any other admissible opinion by a series of minimal changes in the corresponding rankings while staying in the domain at each step. This may be viewed as a 'homogeneity' condition which forbids that opinions are clustered around a few 'representative' opinions. Figure 3 illustrates this; it depicts a maximal Condorcet domain on $X=\{a, b, c, d\}$ that satisfies all conditions of Theorem 1b) except semi-connectedness.

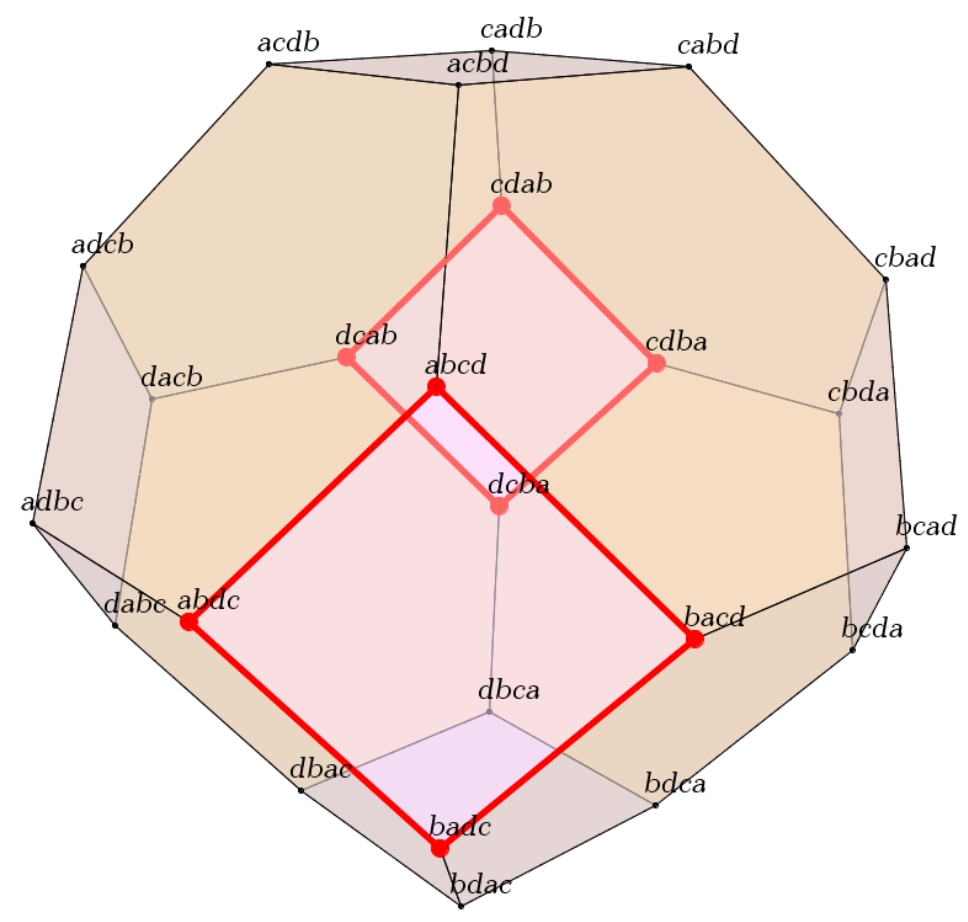

Fig. 3: A non-(semi-)connected, minimally rich Condorcet domain containing pairs of completely reversed orders.

\footnotetext{
${ }^{11}$ The verification of this statement is straightforward if somewhat tedious.
} 
The 'society' corresponding to this domain is clustered around the 'opinion' that the pair of alternatives $\{a, b\}$ dominates the pair $\{c, d\}$ (the 4-cycle in the front) and the opposite 'opinion' that the pair $\{c, d\}$ dominates the pair $\{a, b\}$ (the 4-cycle in the back). That the depicted domain is a maximal Condorcet domain follows again easily using Fact 2.1; that it is not single-peaked follows at once from the fact that it violates the following simple (and well-known) necessary condition for single-peakedness.

Fact 2.2. Suppose that $\mathcal{D} \subseteq \mathcal{P}(X)$ is single-peaked. Then there are at most two alternatives in $X$ which can occur at the bottom of any order in $\mathcal{D} .^{12}$

The conditions of connectedness and also its weakening to semi-connectedness are arguably the most substantial and restrictive conditions used in Theorem 1b) (on top of the consistency of majority voting). Indeed, the minimally rich Condorcet domain displayed in Fig. 3 is only one instance of a general procedure that yields 'large' Condorcet domains that are neither connected nor even semi-connected, Fishburn's so-called replacement scheme (Fishburn [1997]). The scheme takes two Condorcet domains $\mathcal{D}_{1} \subseteq \mathcal{P}\left(X_{1}\right)$ and $\mathcal{D}_{2} \subseteq \mathcal{P}\left(X_{2}\right)$ on two disjoint sets of alternatives and replaces one alternative, say $x \in X_{1}$, in each of the orders in $\mathcal{D}_{1}$ by each of the orders in $\mathcal{D}_{2}$ to obtain a new Condorcet domain $\mathcal{D}_{1} * \mathcal{D}_{2}$ on the set $\left(X_{1} \backslash\{x\}\right) \cup X_{2}$. It is easily verified that the domain $\mathcal{D}_{1} * \mathcal{D}_{2}$ is not semi-connected. On the other hand, $\mathcal{D}_{1} * \mathcal{D}_{2}$ evidently is minimally rich whenever both $\mathcal{D}_{1}$ and $\mathcal{D}_{2}$ are, and it contains two completely reversed orders whenever both $\mathcal{D}_{1}$ and $\mathcal{D}_{2}$ do. Whether the replacement scheme is important for economic applications is open to debate.

\subsubsection{Minimal richness}

Minimal richness has a straightforward interpretation as well: no alternative should a priori be ruled as the individually most desired choice. The condition is termed 'minimal' here because in the literature much stronger 'richness' conditions have been imposed. ${ }^{13}$ Note that the domain of all single-peaked preferences with respect to some fixed linear order $>$ on $X$ in fact also satisfies a stronger richness condition, namely that each alternative occurs not only sometimes as the best but also as the second-best alternative.

Despite its innocuous appearance, the minimal richness condition has quite some bite as well, as illustrated by the two domains depicted in Figure 4. Both domains are maximal, connected Condorcet domains and contain the pair abcd and $d c b a$ of completely reversed orders. Evidently, neither domain is minimally rich, and by Fact 2.2 above, neither domain is single-peaked. That the depicted domains are indeed Condorcet domains follows again from Fact 2.1, and their respective maximality can be verified in a straightforward way.

Interestingly, among all maximal connected Condorcet domains on $X=\{a, b, c, d\}$ with maximal width, the domain on the left hand side of Fig. 4 has the minimal number

\footnotetext{
${ }^{12}$ Note that this condition is clearly not sufficient for single-peakedness as the domain depicted in Fig. 2 shows.

${ }^{13}$ Our terminology follows Aswal et al. [2003] and Chatterji and Sen [2011]; the latter work also discusses domains violating the minimal richness requirement. Stronger richness conditions have been used, e.g., in Chatterji et al. [2013], Nehring and Puppe [2007]).
} 
of elements (in this case, $\# X \cdot(\# X-1) / 2+1=4 \cdot 3 / 2+1=7)$ and the domain on the right hand side has the maximal number of elements. In fact, the domain depicted on the r.h.s. of Fig. 4 has the maximal number of elements among all Condorcet domains on a set of four alternatives, namely 9 (Monjardet [2009]). A Condorcet domain with the maximal number of elements is sometimes referred to as a maximum Condorcet domain. It is known that for $\# X \leq 6$, the maximal number of elements of a Condorcet domain is attained by connected domains with maximal width, ${ }^{14}$ and that the maximal number of elements of such domains always exceeds the number of elements of any single-peaked domain (Monjardet [2009]). Theorem 1b) thus implies that these domains are never minimally rich, and that the maximal cardinality of a semi-connected and minimally rich Condorcet domain is $2^{\# X-1}$, the number of all single-peaked orders with respect to some fixed linear order of $X$.
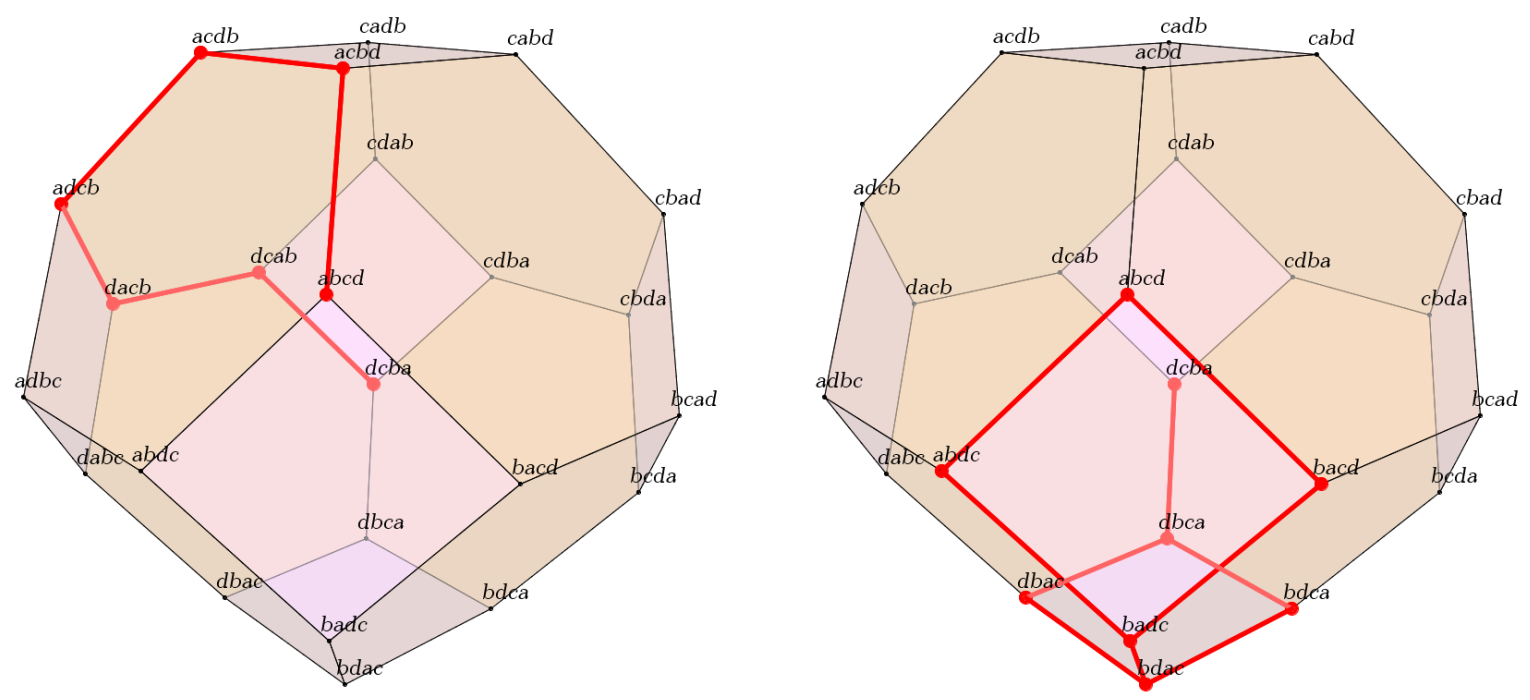

Fig. 4: Two connected but not minimally rich Condorcet domains containing a pair of completely reversed orders.

The strength of the minimal richness condition, at least when imposed jointly with semi-connectedness, can also be inferred from the following immediate corollary of Theorem 1b) and Fact 2.2.

Corollary 2. Let $\# X \geq 3$. There does not exist a semi-connected and minimally rich Condorcet domain on $X$ such that every alternative in $X$ is worst for some order in $\mathcal{D}$.

Note that the domain depicted in Fig. 3 above satisfies all conditions in Corollary 2 except for the semi-connectedness.

\footnotetext{
${ }^{14}$ For $\# X=3,4,5,6$, the maximum Condorcet domains are connected and have 4, 9, 20, 45 elements, respectively (Fishburn $[1997,2002]$ ). For $\# X=7$, the maximal number of elements of a connected Condorcet domain is 100 (Galambos and Reiner [2008]), but it is not known whether this is also the maximal number of elements among all Condorcet domains on a set with 7 elements.
} 


\subsection{Proof of the main result}

First, we show that the domain $\mathcal{S P}_{>}(X)$ of all single-peaked orders with respect to any spectrum $>$ is connected, see also Obraztsova et al. [2013]. ${ }^{15}$ The other properties of $\mathcal{S} \mathcal{P}_{>}(X)$ asserted in Theorem 1a) are obvious.

Thus consider the domain $\mathcal{S P}_{>}(X)$ on $X$ with $a>b>c>\ldots$. We proceed by induction over $\# X$; for the case $\# X=3$ the assertion is obvious. Thus assume that the assertion holds for all cardinalities $<\# X$. Take any two single-peaked orders $P$ and $Q$ in $\mathcal{S P}_{>}(X)$. By moving the alternative $a$ in each of these orders stepwise down to the bottom we obtain two orders $P_{a}$ and $Q_{a}$ that agree with $P$ and $Q$, respectively, on $X \backslash\{a\}$. Since $a$ is an extreme point of the spectrum $>$, the resulting orders at each step as well as $P_{a}$ and $Q_{a}$ are all single-peaked. Thus, $P$ and $P_{a}$, as well as $Q$ and $Q_{a}$ are connected by a path in $\mathcal{S P}_{>}(X)$, respectively. (Note that we allow for the possibility that $P=P_{a}$ and $Q=Q_{a}$.) Now consider the restrictions $\left.P\right|_{X-a}$ and $\left.Q\right|_{X-a}$ of $P$ and $Q$ to the set $X \backslash\{a\}$. Clearly, $\left.P\right|_{X-a}$ and $\left.Q\right|_{X-a}$ are single-peaked on $X \backslash\{a\}$ with respect to the restricted spectrum $b>c>\ldots$ By the induction hypothesis, there exists a path $\left\{P^{1}, P^{2}, \ldots, P^{k}\right\}$ of single-peaked orders on $X \backslash\{a\}$ that connects $\left.P\right|_{X-a}$ and $\left.Q\right|_{X-a}$ such that $P^{1}=\left.P\right|_{X-a}$ and $P^{k}=\left.Q\right|_{X-a}$. For each $j$, denote by $P_{a}^{j}$ the order on $X$ that coincides with $P^{j}$ on $X \backslash\{a\}$ and that puts alternative $a$ at the bottom. Evidently, (i) $P_{a}^{1}=P_{a}$ and $P_{a}^{k}=Q_{a}$, (ii) each order $P_{a}^{j}$ is single-peaked on $X$ with respect to $>$, and (iii) the set $\left\{P_{a}^{1}, P_{a}^{2}, \ldots, P_{a}^{k}\right\}$ constitutes a path in $\mathcal{S P}_{>}(X)$. Combining the path connecting $P$ and $P_{a}$ with the path connecting $P_{a}$ and $Q_{a}$ and the path connecting $Q_{a}$ and $Q$, we obtain a concatenated path connecting $P$ and $Q$, as desired. This proves part a) of Theorem 1 .

The proof of Theorem 1b) starts with the following simple observation. For every $\mathcal{D} \subseteq \mathcal{P}(X)$ and all triples $\{x, y, z\} \subseteq X$ of pairwise distinct alternatives, denote by $\mathcal{D}_{\{x, y, z\}} \subseteq \mathcal{P}(\{x, y, z\})$ the domain of the restrictions of all orders $P \in \mathcal{D}$ to the set $\{x, y, z\}$, i.e.

$$
\mathcal{D}_{\{x, y, z\}}:=\left\{\left.P\right|_{\{x, y, z\}}: P \in \mathcal{D}\right\} .
$$

Fact 2.3. Let $\mathcal{D} \subseteq \mathcal{P}(X)$ and consider any triple $\{x, y, z\} \subseteq X$ of pairwise distinct alternatives.

a) If $\mathcal{D}$ is a Condorcet domain on $X$, then $\mathcal{D}_{\{x, y, z\}}$ is a Condorcet domain on $\{x, y, z\}$.

b) If $\mathcal{D}$ is connected on $X$, then $\mathcal{D}_{\{x, y, z\}}$ is connected on $\{x, y, z\}$.

c) If $\mathcal{D}$ has maximal width on $X$, then $\mathcal{D}_{\{x, y, z\}}$ has maximal width on $\{x, y, z\}$.

d) If $\mathcal{D}$ is semi-connected on $X$, then $\mathcal{D}_{\{x, y, z\}}$ is semi-connected on $\{x, y, z\}$.

e) If $\mathcal{D}$ is minimally rich on $X$, then $\mathcal{D}_{\{x, y, z\}}$ is minimally rich on $\{x, y, z\}$.

Proof. Part a) follows at once from Fact 2.1 iv) above, since a Condorcet cycle in $\mathcal{D}_{\{x, y, z\}}$ would induce a corresponding Condorcet cycle in $\mathcal{D}$. Part b) also follows easily. Consider

\footnotetext{
${ }^{15}$ Theorem 4.10 in Obraztsova et al. [2013] asserts the same result; a proof (different from the one presented here) is available in the full (working paper) version of Obraztsova et al. [2013]. I am grateful to Edith Elkind for notifying me.
} 
any two orders $\tilde{P}$ and $\tilde{Q}$ in $\mathcal{D}_{\{x, y, z\}}$, and let $P, Q \in \mathcal{D}$ be such that $\tilde{P}=\left.P\right|_{\{x, y, z\}}$ and $\tilde{Q}=\left.P^{\prime}\right|_{\{x, y, z\}}$. By assumption there exists a path $\left\{P^{1}, \ldots, P^{k}\right\}$ connecting $P$ and $Q$ in $\mathcal{D}$. Evidently, then $\left\{\left.P^{1}\right|_{\{x, y, z\}}, \ldots,\left.P^{k}\right|_{\{x, y, z\}}\right\}$ is a path in $\mathcal{D}_{\{x, y, z\}}$ that connects $\tilde{P}$ and $\tilde{Q}$ (observe that $\left.P^{j}\right|_{\{x, y, z\}}$ and $\left.P^{l}\right|_{\{x, y, z\}}$ can coincide for distinct $j, l \in\{1, \ldots, k\}$ ). To verify part c), simply observe that if $\bar{P}$ and $\underline{P}$ are completely reversed in $\mathcal{D}$, then $\left.\bar{P}\right|_{\{x, y, z\}}$ and $\left.\underline{P}\right|_{\{x, y, z\}}$ are completely reversed in $\mathcal{D}_{\{x, y, z\}}$. Part d) follows at once from parts b) and c). Finally, if $\mathcal{D}$ is minimally rich, then in particular $x, y$ and $z$ must be on top of at least order in $\mathcal{D}$; this proves part e).

The significance of the previous result in our context stems from the observation that on a set of three alternatives, there are only two types of semi-connected Condorcet domains: the single-peaked and the 'single-dipped' domain (as also observed by [Danilov et al., 2012, Th. 4(ii)]). A domain $\mathcal{D} \subseteq \mathcal{P}(X)$ is called single-dipped with respect to the linear order $>$ on $X$ if, for all $P \in \mathcal{D}$ and all $w \in X$, the lower contour sets $L_{P}(w):=\{y \in$ $X: w P y\}$ are connected ('convex') in the order $>$, i.e. $\{x, z\} \subseteq L_{P}(w)$ and $x<y<z$ jointly imply $y \in L_{P}(w)$. A domain $\mathcal{D}$ is called single-dipped if there exists some linear order $>$ such that $\mathcal{D}$ is single-peaked with respect to $>.{ }^{16}$ The domain of all orders that are single-dipped with respect to the fixed order $>$ on $X$ is denoted by $\mathcal{S D}(X)$.

Fact 2.4. There are exactly two semi-connected Condorcet domains on the set $\{x, y, z\} \subseteq$ $X$ that contain the two completely reversed orders $x y z$ and $z y x$, namely

$$
\begin{aligned}
& \mathcal{S P}_{>}(\{x, y, z\})=\{x y z, y x z, y z x, z y x\} \text {, and } \\
& \mathcal{S D}_{>}(\{x, y, z\})=\{x y z, x z y, z x y, z y x\} \text {, }
\end{aligned}
$$

where the spectrum $>$ is given either by the order $x y z$, or by the completely reversed order zyx (see Figure 5).
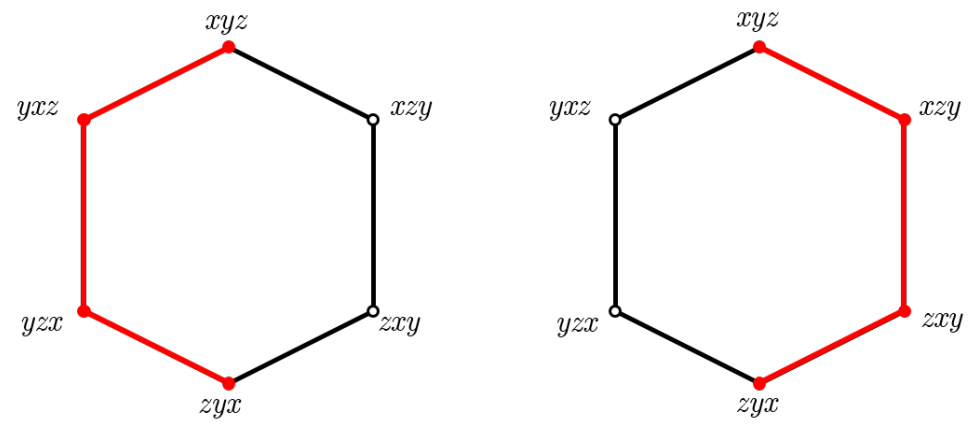

Fig. 5: The single-peaked (left) and single-dipped (right) domains on $\{x, y, z\}$.

Proof. By the semi-connectedness of $\mathcal{D}$, there exists a path in $\mathcal{D}$ connecting $x y z$ and $z y x$. Thus, $\mathcal{D}$ contains at least one neighbor of $x y z$, i.e. either $y x z$ or $x z y$. On the other

\footnotetext{
${ }^{16}$ Inada [1964] introduced the condition of single-dippedness under the name of 'single-cavedness.' We follow here the terminology of Klaus et al. [1997] and others.
} 
hand, $\mathcal{D}$ cannot contain both of these neighbors because the triple $y x z, x z y, z y x$ forms a Condorcet cycle. If $\mathcal{D}$ contains $y x z$ it must coincide with $\mathcal{S} \mathcal{P}_{>}(\{x, y, z\})$ by the semiconnectedness; similarly, if it contains $x z y$ it must coincide with $\mathcal{S D}_{>}(\{x, y, z\})$.

Remark. By Fact 2.4, semi-connectedness of a Condorcet domain implies its connectedness in the case of three alternatives. This does not hold in general. However, in the appendix it is shown that every semi-connected and closed Condorcet domain must in fact be connected; thus, for closed (in particular, for all maximal) Condorcet domains with maximal width the notions of semi-connectedness and connectedness coincide, see Propositions A.1 and A.2.

We can now complete the proof of Theorem $1 \mathrm{~b})$. Thus, suppose that $\mathcal{D}$ is semiconnected and minimally rich. By the semi-connectedness there exist two completely reversed orders $\bar{P}, \underline{P} \in \mathcal{D}$ that are connected by a path in $\mathcal{D}$. By the minimal richness, every alternative is in top of some order in $\mathcal{D}$. Consider any triple $x, y, z \in X$ with $x \bar{P} y \bar{P} z$. By Fact 2.3, the domain $\mathcal{D}_{\{x, y, z\}}$ is minimally rich and semi-connected. Since the single-dipped domain $\mathcal{S D}_{>}(\{x, y, z\})$ is not minimally rich, we obtain by Fact 2.4 that, for all triples $x, y, z \in X$,

$$
\mathcal{D}_{\{x, y, z\}}=\mathcal{S} \mathcal{P}_{>}(\{x, y, z\}),
$$

where $>$ is given by the restriction of $\bar{P}$ to $\{x, y, z\}$. Thus, the restriction of $\mathcal{D}$ to any triple is single-peaked with respect to the spectrum inherited from $\bar{P}$ (or, equivalently, from $\underline{P})$.

It remains to show that this implies the single-peakedness of $\mathcal{D}$ itself. This is the content of the following simple observation.

Fact 2.5. Let $\mathcal{D}$ satisfy (2.1) for all triples $x, y, z \in X$ where $>$ is given by the restriction of $\bar{P}$ to $\{x, y, z\}$. Then $\mathcal{D}$ is single-peaked with respect to $>=\bar{P}$.

Proof. We have to show that all upper contour sets of all orders $P \in \mathcal{D}$ are connected in the order $>=\bar{P}$. Thus, consider any order $P \in \mathcal{D}$ and any triple $x, y, z$ with $x>y>z$, i.e. $x \bar{P} y \bar{P} z$, and suppose that $\{x, z\} \subseteq U_{P}(w)$ for some $w \in X$. By (2.1) the restriction of $P$ to $\{x, y, z\}$ takes one of the following form: $x P y P z, y P x P z, y P z P x$, or $z P y P x$ (cf. Fig. 5). Evidently, in each case $\{x, z\} \subseteq U_{P}(w)$ implies $y \in U_{P}(w)$.

\subsection{A dual characterization of the single-dipped domain}

Theorem 1 above entails a 'dual' characterization of the single-dipped domain in a straightforward way, as follows.

Theorem 2. a) For every linear order $>$ on $X$, the domain $\mathcal{S D}_{>}$is a connected Condorcet domain with maximal width such that every alternative in $X$ is the worst alternative for some order.

b) Conversely, let $\mathcal{D} \subseteq \mathcal{P}(X)$ be a semi-connected Condorcet domain such that every alternative in $X$ is the worst alternative for some order in $\mathcal{D}$. Then, $\mathcal{D}$ is singledipped. 
Proof. Part a) is analogous to the proof of Theorem 1a). For part b) note that among the two admissible semi-connected domains on triples according to Fact 2.4 only the singledipped domain has every alternative at the bottom of at least one order. Thus, if $\mathcal{D}$ satisfies the conditions of Theorem $2 \mathrm{~b}$ ), and if $\bar{P} \in \mathcal{D}$ is one of a pair of completely reversed orders in $\mathcal{D}$ that are connected by a path in $\mathcal{D}$, we obtain, for all triples $x, y, z \in X$,

$$
\mathcal{D}_{\{x, y, z\}}=\mathcal{S D}_{>}(\{x, y, z\}),
$$

where $>$ is given by the restriction of $\bar{P}$ to $\{x, y, z\}$. By an argument completely symmetric to that given in Fact 2.5 we obtain from (2.2) that the lower contour sets of all orders in $\mathcal{D}$ are connected in the spectrum $>=\bar{P}$, and thus that $\mathcal{D}$ itself is single-dipped.

\subsection{A corollary for single-crossing domains}

Our analysis has an important implication for a class of domains known as 'single-crossing' domains. A domain $\mathcal{D} \subseteq \mathcal{P}(X)$ is a single-crossing domain if it can be written in the form $\mathcal{D}=\left\{P_{1}, \ldots, P_{m}\right\}$ such that, for all ordered pairs $(x, y) \in X \times X$, the set $\{P \in$ $\mathcal{D}: x P y\}$ is 'connected' in $\{1, \ldots, m\}$, i.e., for all $x, y \in X, x P_{j} y$ and $x P_{l} y$ with $j<l$ implies $x P_{k} y$ for all $k \in\{j, \ldots, l\}$, and $y P_{j} x$ and $y P_{l} x$ with $j<l$ implies $y P_{k} x$ for all $k \in\{j, \ldots, l\}$. This property has been introduced in the literature by Rothstein [1990] under the name of 'order-restriction.' It underlies the analysis in Roberts [1977], Gans and Smart [1996], and is employed frequently under the name of 'single-crossing property' (see, e.g., Saporiti [2009]). It is well-known that all single-crossing domains are Condorcet domains (Rothstein [1990, 1991]).

Corollary 3. Let $\mathcal{D} \subseteq \mathcal{P}(X)$ be a single-crossing domain. If $\mathcal{D}$ is minimally rich, then it is single-peaked. If every alternative in $X$ is worst in at least one order in $\mathcal{D}$, then $\mathcal{D}$ is single-dipped.

Proof. Every single-crossing domain $\mathcal{D}$ can be extended to a semi-connected singlecrossing domain $\mathcal{D}^{*} \supseteq \mathcal{D}$ (note that we are not asserting that $\mathcal{D}^{*}$ is a maximal Condorcet domain). Indeed, if $\mathcal{D}=\left\{P_{1}, \ldots, P_{k}\right\}$ has the single-crossing property, then so does the domain $\left\{P_{1}, \ldots, P_{k}, P_{k+1}\right\}$ where $P_{k+1}=P_{1}^{\text {inv }}$ (we allow that $P_{k+1}=P_{k}$ ). We can now fill possible 'gaps' in the sequence $P_{1}, \ldots, P_{k}, P_{k+1}$ as follows. If $P_{1}$ and $P_{2}$ differ in the ranking of more than one pair of alternatives, at least one of these pairs must be an adjacent pair in $P_{1}$. Then, we can add the order $P_{1}^{\prime}$ that switches exactly this pair and agrees with $P_{1}$ in the ranking of all other pairs, and consider the domain $\left\{P_{1}, P_{1}^{\prime}, P_{2}, \ldots\right\}$. Continuing in this fashion, we obtain a semi-connected and single-crossing domain $\mathcal{D}^{*} \supseteq \mathcal{D}$. Evidently, if $\mathcal{D}$ is minimally rich, so is $\mathcal{D}^{*}$; and if every alternative is worst in at least one order in $\mathcal{D}$, then the same property holds for $\mathcal{D}^{*}$. Since every single-crossing domain is a Condorcet domain, we thus obtain by Theorem $1 b$ ) that $\mathcal{D}^{*}$, and hence also $\mathcal{D}$, is single-peaked if $\mathcal{D}$ is minimally rich. Similarly, if $\mathcal{D}$ has every alternative at the bottom of some order, then $\mathcal{D}^{*}$, and hence also $\mathcal{D}$, is single-dipped by Theorem $2 \mathrm{~b}$ ). 
Remark. The semi-connected domain $\mathcal{D}^{*}$ constructed in the proof of Corollary 3 is in fact a maximal single-crossing domain in the sense that no proper superdomain of $\mathcal{D}^{*}$ can be single-crossing. The maximal single-crossing domains are sometimes referred to as maximal chains in the literature, since they indeed correspond to the maximal chains in the so-called 'weak Bruhat order' (Abello [1991], Chameni-Nembua [1989], Galambos and Reiner [2008], Monjardet [2009]). Note that the maximal single-crossing domains are in general not maximal as Condorcet domains. For instance, all paths connecting the orders $a b c d$ and $d c b a$ in the single-peaked domain in Fig. 1, as well as in the maximum domain on the right hand side of Fig. 4 correspond to maximal single-crossing domains; but evidently, these maximal paths do not form maximal Condorcet domains. On the other hand, the maximal single-crossing domain on the left hand side of Fig. 4 is also maximal as Condorcet domain; a simple necessary and sufficient condition for the maximality (as Condorcet domain) of a maximal single-crossing domain is given in [Monjardet, 2007, p. 79] and Puppe and Slinko [2015]. The fact that every single-crossing profile of linear orders in which every alternative is at the top of at least one voter must be single-peaked has also been observed by Elkind et al. [2014].

\section{The case of weak preference orders}

\subsection{The basic concepts and definitions generalized}

Consider now the set $\mathcal{R}(X)$ of all weak orders (i.e., complete and transitive binary relations) on $X$, and subdomains $\mathcal{D} \subseteq \mathcal{R}(X)$. Individual weak preferences are denoted by $R, R^{\prime}$ etc., and profiles by $\rho=\left(R_{1}, \ldots, R_{n}\right) \in \mathcal{R}(X)^{n}$. Frequently, we will denote weak orders by listing the alternatives in descending order and putting indifferent alternatives in brackets, e.g. the weak order that ranks $a$ first and $b$ and $c$ indifferently as second best is denoted by $a(b c) \ldots$; similarly, the weak order that has $a$ and $b$ indifferently at the top and $c$ at the following rank is denoted by $(a b) c \ldots$; finally, the weak order that has $a, b$ and $c$ indifferently on top is denoted by $(a b c) \ldots$, etc.

The majority relation associated with a profile $\rho$ is the binary relation $R_{\rho}^{\operatorname{maj}}$ on $X$ such that $x R_{\rho}^{\mathrm{maj}} y$ if and only if $x R_{i} y$ for more than half of the voters. ${ }^{17}$ Note that, according to this definition, the majority relation is complete for every odd profile $\rho$. As above, the domains $\mathcal{D} \subseteq \mathcal{R}(X)$ such that, for all odd $n$, the majority relation associated with any profile $\rho \in \mathcal{D}^{n}$ is transitive are referred to as Condorcet domains. Condorcet domains of weak orders have been studied much less than their counterparts with linear orders, see the monograph of Gaertner [2001] for a state-of-the-art survey. As above, a domain $\mathcal{D} \subseteq \mathcal{R}(X)$ is called a maximal Condorcet domain (of weak orders) if every Condorcet domain (on the same set of alternatives) that contains $\mathcal{D}$ as a subset must in fact coincide with $\mathcal{D}$.

\footnotetext{
${ }^{17}$ It is well-known that this is not the only possible definition of majority rule with weak preferences (see, e.g., [Gaertner, 2001, Ch. 3]); for our purpose it turns out to be the appropriate one. Moreover, it represents the natural notion of majority rule inherited from the general judgement aggregation model, see List and Puppe [2009], Nehring et al. [2014, forthcoming].
} 
The generalization of our main result to the case of weak orders requires careful adaption of the employed concepts since some additional complications arise. As a first indication of these, observe that the equivalences in Fact 2.1 do not carry over to the domain of weak orders. As a simple example, consider the domain on $X=\{a, b, c\}$ consisting of the three weak orders $(a b) c, a(b c)$ and $c b a$, say. If $\rho$ is the profile in which three voters have each one of these preferences, respectively, the majority relation is acyclic but not transitive, since a majority strictly prefers $a$ to $c$ while $\left[a R_{\rho}^{\text {maj }} b\right.$ and $\left.b R_{\rho}^{\text {maj }} a\right]$ as well as $\left[b R_{\rho}^{\mathrm{maj}} c\right.$ and $\left.c R_{\rho}^{\mathrm{maj}} b\right]$, i.e. both pairs of alternatives $(a, b)$ and $(b, c)$ are deemed indifferent, respectively, according to the majority relation. Thus, the domain is not a Condorcet domain in our sense.

A domain $\mathcal{D} \subseteq \mathcal{R}(X)$ is called weakly single-peaked with respect to the linear order $>$ on $X$ if, for all $R \in \mathcal{D}$ and all $w \in X$, the upper contour sets $U_{R}(w):=\{y \in X: y R w\}$ are connected ('convex') in the order $>$, i.e. $\{x, z\} \subseteq U_{R}(w)$ and $x<y<z$ jointly imply $y \in U_{R}(w)$. A domain $\mathcal{D} \subseteq \mathcal{R}(X)$ is called weakly single-peaked if there exists some linear order $>$ such that $\mathcal{D}$ is weakly single-peaked with respect to $>$. Similarly, a domain $\mathcal{D} \subseteq \mathcal{R}(X)$ is called weakly single-dipped if there exists a linear order $>$ on $X$ such that, for all $R \in \mathcal{D}$, the lower contour sets $L_{R}(w):=\{y \in X: w R y\}$ are connected with respect to $>$.

Remark. Several generalizations of the concept of single-peakedness in order to accommodate indifference have been discussed in the literature. [Moulin, 1988, p. 264] notes that for many purposes indifferences across the (unique) peak could be allowed without difficulty. On the other hand, the concept of 'single-plateaued' preferences allows for multiple best alternatives and has proven useful in some contexts ([Gaertner, 2001, p. 68]); however, this concept still assumes strict monotonicity below the optimum (Moulin [1984]). The above notion of 'weakly' single-peaked preferences has also been employed by Duggan [2016] (under this name) and is weaker than single-plateauedness as it allows for multiple 'plateaus' and, in particular, not only at the top. From an abstract perspective, it represents a natural generalization of the usual notion of single-peakedness, as it corresponds to convexity of all upper contour sets of the weak relations, just as standard single-peakedness corresponds to convexity of the upper contour sets of all strict relations. However, I do not want to argue that the adopted definitions are the only reasonable extensions of the concepts of single-peakedness and Condorcet domain to the case of weak orders. For instance, one could require only acyclicity of the majority relation for a domain to be called 'Condorcet domain,' or base the notion of Condorcet domain on the so-called 'strict' majority relation ([Gaertner, 2001, p. 27]), or require uniqueness of the top alternative in the definition of single-peakedness. However, as the subsequent analysis will show, the adopted definitions are precisely the appropriate notions for which the robustness of the main characterization result can be demonstrated.

A further important difference to the case of linear orders is that not all weakly singlepeaked domains are Condorcet domains; in particular, the domain of all weak orders that are weakly single-peaked with respect to some order $>$ is not a Condorcet domain. The same example as above can be used to demonstrate this. Indeed, we have already argued 
above that the domain $\mathcal{D}=\{(a b) c, a(b c), c b a\}$ is not a Condorcet domain. On the other hand, it is clearly weakly single-peaked with respect to the linear order $a>b>c$.

We also have to generalize the notion of 'connectedness,' as follows. For $R \in \mathcal{R}(X)$, denote by $\neg R$ the negation of $R$, i.e., for all $x, y \in X, x \neg R y \Leftrightarrow \operatorname{not} x R y$. For any two orders $R, R^{\prime} \in \mathcal{R}(X)$, denote by $\left[R, R^{\prime}\right] \subseteq \mathcal{R}(X)$,

$$
\left[R, R^{\prime}\right]:=\left\{Q \in \mathcal{R}(X): Q \supseteq R \cap R^{\prime} \text { and } \neg Q \supseteq \neg R \cap \neg R^{\prime}\right\} .
$$

We will refer to $\left[R, R^{\prime}\right]$ as the interval spanned by $R$ and $R^{\prime}$, and to its elements as the orders between $R$ and $R^{\prime}$; furthermore, two distinct orders $R$ and $R^{\prime}$ are called $\mathcal{R}(X)$-neighbors if $\left[R, R^{\prime}\right]=\left\{R, R^{\prime}\right\}$. Figure 6 depicts the 'neighborhood structure' of $\mathcal{R}(\{a, b, c\})$, connecting all neighbors by an edge. Note that, due to transitivity of the indifference relation, the six neighbors of the weak order $(a b c)$ on the set $X=\{a, b, c\}$ each differ from $(a b c)$ in the ranking of two pairs of alternatives, respectively. ${ }^{18}$

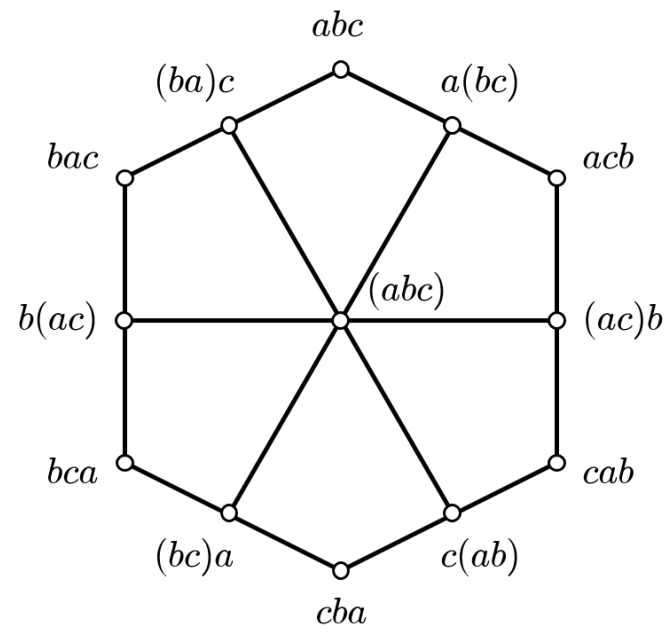

Fig. 6: The neighborhood structure of $\mathcal{R}(\{a, b, c\})$.

A path in $\mathcal{R}(X)$ is a subset $\left\{R_{1}, \ldots, R_{m}\right\} \subseteq \mathcal{R}(X)$ with $m \geq 2$ such that for all $j=1, \ldots, m-1$, the two consecutive orders $R_{j}$ and $R_{j+1}$ are $\mathcal{R}(X)$-neighbors. A domain $\mathcal{D} \subseteq \mathcal{R}(X)$ will be called connected if, for every pair $R, R^{\prime} \in \mathcal{D}$ of distinct orders in $\mathcal{D}$, there exists a path $\left\{R_{1}, \ldots, R_{m}\right\}$ that connects $R$ and $R^{\prime}$ (i.e. $R_{1}=R$ and $R_{m}=R^{\prime}$ ) and that lies entirely in $\mathcal{D}$ (i.e. $R_{j} \in \mathcal{D}$ for all $j=1, \ldots, m$ ).

As above, two orders $R$ and $R^{\text {inv }}$ are called completely reversed if, for all distinct $x$ and $y, x R y \Leftrightarrow \operatorname{not}\left(x R^{\text {inv }} y\right)$. Note that by the completeness assumption, two completely reversed weak orders must both in fact be linear orders, i.e. neither of the two can contain any non-trivial indifference. A domain $\mathcal{D} \subseteq \mathcal{R}(X)$ is said to have maximal width if $\mathcal{D}$

\footnotetext{
${ }^{18}$ The neighborhood relation can be defined analogously on the space $\mathcal{P}(X)$ of all linear orders; in this case, definition (3.1) simplifies to $\left[P, P^{\prime}\right]=\left\{Q \in \mathcal{P}(X): Q \supseteq P \cap P^{\prime}\right\}$ due to the antisymmetry condition. Moreover, two linear orders are $\mathcal{P}(X)$-neighbors in $\mathcal{P}(X)$ if and only if they differ in the ranking of exactly one pair of alternatives (see the appendix for further details).
} 
contains at least one pair of completely reversed orders. Finally, a domain $\mathcal{D} \subseteq \mathcal{R}(X)$ is called semi-connected if $\mathcal{D}$ contains at least two completely reversed orders and an entire path connecting them.

\subsection{All (semi-)connected Condorcet domains on triples}

As in the case of linear orders, a crucial step in our characterization of the single-peaked (and single-dipped) domain will be the complete classification of the semi-connected Condorcet domains on triples. This is provided by the next result (see Figure 7 for illustration with the domains $\mathcal{D}_{1}-\mathcal{D}_{6}$ depicted clockwise from top left in the first row to bottom left in the second row).

Proposition 3.1. Let $x, y, z \in X$ be pairwise distinct. The following are the semiconnected Condorcet subdomains of $\mathcal{R}(\{x, y, z\})$ that contain the completely reversed orders xyz and zyx. All of them are maximal and in fact connected.

$$
\begin{aligned}
& \mathcal{D}_{1}(x, y, z)=\{x y z,(x y) z, y x z, y(x z), y z x,(y z) x, z y x\} \\
& \mathcal{D}_{2}(x, y, z)=\{x y z,(x y) z,(x y z), z(x y), z y x\} \\
& \mathcal{D}_{3}(x, y, z)=\{x y z,(x y) z,(x y z),(y z) x, z y x\}, \\
& \mathcal{D}_{4}(x, y, z)=\{x y z, x(y z),(x y z), z(x y), z y x\}, \\
& \mathcal{D}_{5}(x, y, z)=\{x y z, x(y z),(x y z),(y z) x, z y x\}, \\
& \mathcal{D}_{6}(x, y, z)=\{x y z, x(y z), x z y,(x z) y, z x y, z(x y), z y x\} .
\end{aligned}
$$

Proof. By the semi-connectedness of $\mathcal{D}$, there exists a path in $\mathcal{D}$ connecting $x y z$ and $z y x$. Thus, $\mathcal{D}$ contains at least one neighbor of $x y z$, i.e. either $(x y) z$ or $x(y z)$. On the other hand, $\mathcal{D}$ cannot contain both of these neighbors because the triple $[(x y) z, x(y z)$, $z y x]$ is 'forbidden:' if three voters each have one of these preference orders, respectively, we obtain a non-transitive majority relation since $x$ and $y$, as well as $y$ and $z$ are deemed indifferent by the majority relation while $x$ is strictly superior to $z$.

Case 1. Suppose first that $\mathcal{D}$ contains $(x y) z$. By the semi-connectedness, $\mathcal{D}$ must contain either the neighbor $y x z$, or the neighbor $(x y z)$ of $(x y) z$ 'in direction of' $z y x$. Again, $\mathcal{D}$ cannot contain both of them since the triple $[y x z,(x y z), z y x]$ is forbidden (in the same sense as above).

Case 1.1. Thus, suppose $\mathcal{D}$ contains yxz. By the semi-connectedness it must contain $\mathcal{D}_{1}(x, y, z)$. On the other hand, $\mathcal{D}_{1}(x, y, z)$ is a maximal Condorcet domain. For this it remains to show that it cannot contain any order from the set $\{x z y,(x z) y, z x y, z(x y)\}$. For the two linear orders $x z y$ and $z x y$ this follows immediately from the existence of appropriate Condorcet cycles with two elements from $\mathcal{D}_{1}(x, y, z)$, respectively; for the order $z(x y)$ it follows since the triple $[x y z,(y z) x, z(x y)]$ is forbidden; and for $(x z) y$ it follows since the triple $[(x y) z, y z x,(x z) y]$ is forbidden. Thus, in Case 1.1 we obtain $\mathcal{D}=\mathcal{D}_{1}(x, y, z)$.

Case 1.2. Now suppose $\mathcal{D}$ contains $(x y z)$ (in addition to $(x y) z$ ). Then, $\mathcal{D}$ can neither contain $y(x z)$ nor $(x z) y$ since both triples $[(x y) z,(x y z), y(x z)]$ and $[x y z,(x y z),(x z) y]$ are 
forbidden. ${ }^{19}$ Thus, by the semi-connectedness $\mathcal{D}$ must contain either $z(x y)$ or $(y z) x$, but as already argued, it cannot contain both. In the first case, $\mathcal{D}$ thus contains $\mathcal{D}_{2}(x, y, z)$, and in the second case $\mathcal{D}$ contains $\mathcal{D}_{3}(x, y, z)$. But by arguments completely symmetric to those given so far it follows that either of these domains is maximal.

Case 2. If $\mathcal{D}$ contains $x(y z)$, a completely symmetric analysis yields the possible cases $\mathcal{D}=$ $\mathcal{D}_{4}(x, y, z), \mathcal{D}=\mathcal{D}_{5}(x, y, z)$, or $\mathcal{D}=\mathcal{D}_{6}(x, y, z)$. This completes the proof of Proposition 3.1 .

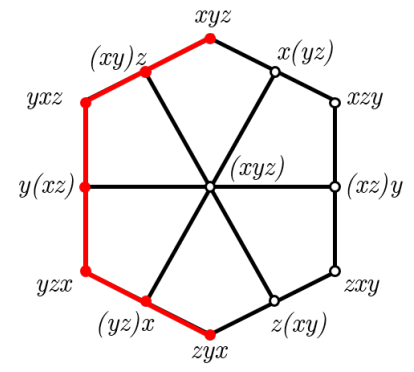

$\mathcal{D}_{1}(x, y, z)$

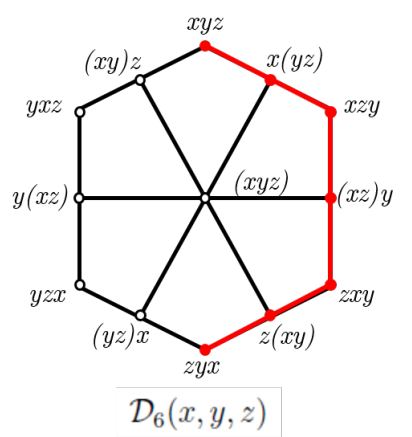

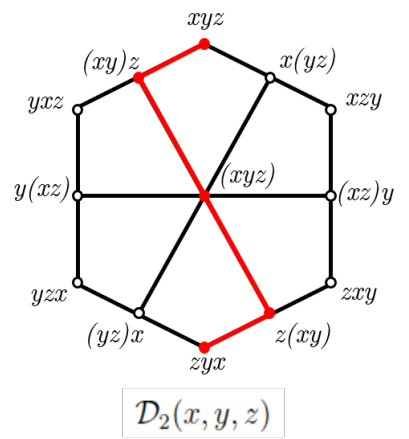

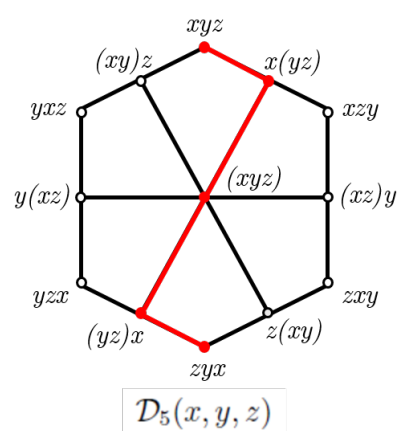

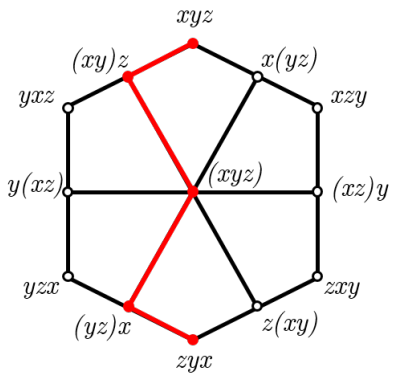

$\mathcal{D}_{3}(x, y, z)$

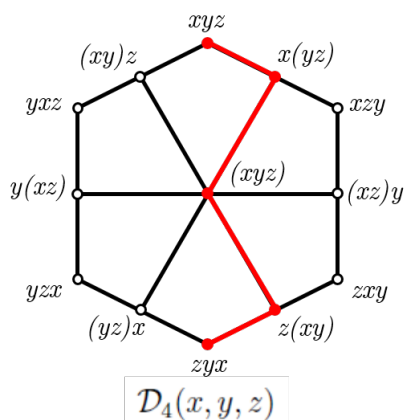

Fig. 7: The (maximal) (semi-)connected Condorcet subdomains of $\mathcal{R}(\{x, y, z\})$.

A crucial observation for the following is that the domains $\mathcal{D}_{1}-\mathcal{D}_{5}$ are weakly singlepeaked, while the domains $\mathcal{D}_{2}-\mathcal{D}_{6}$ are weakly single-dipped with respect to the linear order $x>y>z$ (in particular, the five-element domains $\mathcal{D}_{2}-\mathcal{D}_{5}$ are simultaneously weakly single-peaked and weakly single-dipped with respect to $x>y>z$ ).

\subsection{Main result}

Due to the inclusion of indifferences, there are two natural formulations of the minimal richness condition. Let us say that a domain $\mathcal{D} \subseteq \mathcal{R}(X)$ is strongly minimally rich if, for each alternative $x \in X$, there is at least one weak order $R \in \mathcal{D}$ that has $x$ has the unique top alternative. Analogously, say that a domain $\mathcal{D} \subseteq \mathcal{R}(X)$ is weakly minimally rich if, for

\footnotetext{
${ }^{19}$ That these two triples are forbidden hinges on the adopted definition of the majority relation. For instance, if in a profile $\rho$, each of the orders $[(x y) z,(x y z), y(x z)]$ receives one third of the votes, we obtain both $x R_{\rho}^{\mathrm{maj}} y$ and $y R_{\rho}^{\mathrm{maj}} x$; moreover, $x R_{\rho}^{\mathrm{maj}} z$ and $z R_{\rho}^{\mathrm{maj}} x$ but not $z R_{\rho}^{\mathrm{maj}} y$.
} 
each alternative $x \in X$, there is at least one weak order $R \in \mathcal{D}$ such that $x$ is among the top alternatives of $R$.

The following characterization extends the main result of the previous section to the case of weak orders.

Theorem 3. a) For every linear order $>$ on $X$, there exists a unique maximal Condorcet domain $\hat{\mathcal{R}}_{>} \subseteq \mathcal{R}(X)$ that contains the domain $\mathcal{S P}_{>}(X)$ of all single-peaked linear orders with respect to $>$. The domain $\hat{\mathcal{R}}_{>}$is weakly single-peaked and connected. Evidently, $\hat{\mathcal{R}}_{>}$has maximal width and is strongly minimally rich.

b) Conversely, let $\mathcal{D} \subseteq \mathcal{R}(X)$ be a semi-connected and weakly minimally rich Condorcet domain. Then, $\mathcal{D}$ is weakly single-peaked.

c) Moreover, if $\mathcal{D} \subseteq \mathcal{R}(X)$ is a semi-connected and strongly minimally rich Condorcet domain, then $\mathcal{D} \subseteq \hat{\mathcal{R}}_{>}$for some linear order $>$on $X$. (In particular, $\mathcal{D}$ is weakly single-peaked.)

The domain $\hat{\mathcal{R}}_{>}$admits the following explicit characterization. First, each indifference class of every weak order in $\hat{\mathcal{R}}_{>}$has at most two elements, i.e. any indifference prevails over at most two distinct alternatives. Moreover, whenever all indifferences are 'resolved' for a weak order in $\hat{\mathcal{R}}_{>}$by transforming all indifferent pairs into strictly ranked adjacent alternatives, one obtains an order in $\mathcal{S P}_{>}(X)$, no matter which combination of strict rankings for the indifferent pairs is chosen. For illustration, consider Figure 8 which depicts the domain $\hat{\mathcal{R}}_{>}$on $X=\{a, b, c, d\}$ for the spectrum $a>b>c>d$.

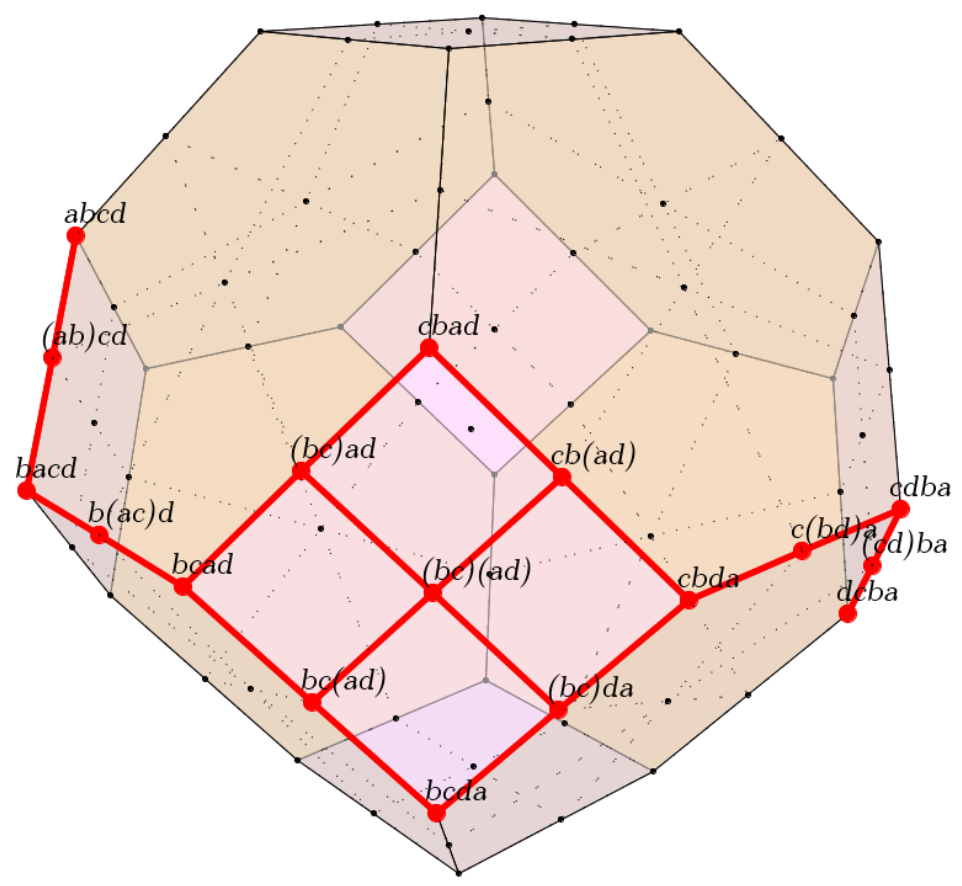

Fig. 8: The domain $\hat{\mathcal{R}}_{>}$on $X=\{a, b, c, d\}$ with spectrum $a>b>c>d$. 
As an example, consider the weak order $(b c)(a d)$ in the middle of Fig. 8, i.e. the order that declares $b$ and $c$ as indifferent on the top and $a$ and $d$ as indifferent at the bottom. There are exactly four ways to transform these two indifferent pairs into adjacent strictly ranked pairs resulting in the linear orders $b c a d, c b a d, b c d a$, and $c b d a$, all of which belong to $\mathcal{S P}_{>}(\{a, b, c, d\})$ for the spectrum $a>b>c>d$. By contrast, the order $a(b c) d$ does not belong to the domain $\hat{\mathcal{R}}_{>}$for the spectrum $a>b>c>d$, since the linear order $a c b d$ is not single-peaked. Note that the weak order $a(b c) d$ is nevertheless weakly single-peaked.

Proof of Theorem 3. a) Let $\mathcal{D} \subseteq \mathcal{R}(X)$ be a maximal Condorcet domain with $\mathcal{D} \supseteq \mathcal{S P}_{>}(X)$ for a given spectrum $>$ on $X$. As is easily verified, for every triple of distinct alternatives $x, y, z \in X$ with $x>y>z$, the restriction $\mathcal{D}_{\{x, y, z\}}$ of $\mathcal{D}$ to $\{x, y, z\}$ is a maximal Condorcet domain of weak orders on $\{x, y, z\}$ that contains the domain $\mathcal{S P}_{>}(\{x, y, z\})$ (strictly speaking, in the latter term ' $>$ ' denotes the restriction of $>$ to $\{x, y, z\})$. By Fact $2.1, \mathcal{D}_{\{x, y, z\}}$ can neither contain the linear order $x z y$ nor the linear order $z x y$. Moreover, $\mathcal{D}_{\{x, y, z\}}$ can contain neither of the weak orders $x(y z),(x z) y, z(x y)$, nor the complete indifference relation $(x y z)$; indeed, the following are forbidden triples of weak orders: $[x(y z), y x z, z y x],[(x z) y, y x z, z y x],[z(x y), x y z, y z x],[(x y z), x y z, y z x]$. This implies that $\mathcal{D}_{\{x, y, z\}} \subseteq \mathcal{D}_{1}(x, y, z)$, where $\mathcal{D}_{1}(x, y, z)$ is defined as in Proposition 3.1 above. By the maximality of both $\mathcal{D}_{\{x, y, z\}}$ and $\mathcal{D}_{1}(x, y, z)$, we obtain that in fact $\mathcal{D}_{\{x, y, z\}}=\mathcal{D}_{1}(x, y, z){ }^{20}$ This implies the desired conclusion $\mathcal{D}=\hat{\mathcal{R}}_{>}$since evidently no indifference class in any order in $\mathcal{D}$ can have more than two elements; moreover, for no weak order in $\mathcal{D}$, one can obtain a linear order outside $\mathcal{S} \mathcal{P}_{>}(X)$ by strictly ranking all indifferent pairs and keeping the relative position of all other pairs of alternatives fixed (indeed, if this was possible there would exist a triple $x>y>z$ such that $\mathcal{D}_{\{x, y, z\}}$ would contain at least one of the weak orders $x(y z),(x z) y$, or $z(x y))$. From the above it is immediate that the domain $\hat{\mathcal{R}}_{>}$is weakly single-peaked. It is easily seen that $\hat{\mathcal{R}}_{>}$is semi-connected: indeed, any two single-peaked orders in $\mathcal{S P}_{>}(X)$ that differ in the ranking of exactly one pair are connected to each other in $\hat{\mathcal{R}}_{>}$by the weak order that declares this pair of alternatives as indifferent (keeping the position of all other alternatives fixed). The connectedness of $\hat{\mathcal{R}}_{>}$ thus follows from Proposition A.2 in the appendix. This completes the proof of part a).

b) Suppose that $\mathcal{D} \subseteq \mathcal{R}(X)$ is a semi-connected and weakly minimally rich Condorcet domain. Denote by $\bar{P}$ one of the pair of orders in $\mathcal{D}$ that are complete inverses of each other and are connected by a path in $\mathcal{D}$ (recall that each one of a pair of completely reversed orders in $\mathcal{R}(X)$ must be a linear order). For each triple of distinct alternatives $x, y, z \in X$ with $x \bar{P} y \bar{P} z$, the restriction $\mathcal{D}_{\{x, y, z\}}$ is a semi-connected and weakly minimally rich Condorcet domain that contains the completely reversed orders $x y z$ and $z y x$. By Proposition 3.1, $\mathcal{D}_{\{x, y, z\}}$ coincides with one of the domains $\mathcal{D}_{1}(x, y, z)-\mathcal{D}_{6}(x, y, z)$, but by the weak minimal richness in fact with one of the domains $\mathcal{D}_{1}(x, y, z)-\mathcal{D}_{5}(x, y, z)$ (observe that no weak order in $\mathcal{D}_{6}(x, y, z)$ has $y$ among its top alternatives). But we have

\footnotetext{
${ }^{20}$ Note that we cannot invoke Proposition 3.1 to directly conclude this, since we do not yet know whether or not $\mathcal{D}$ is semi-connected. In fact, there exist several maximal Condorcet domains of weak orders on a triple $\{x, y, z\}$ different from the ones listed in Proposition 3.1; these domains are either not connected or do not have maximal width (cf. Dittrich [2016]). The argument just given in the main text shows that none of them contains the single-peaked domain $\mathcal{S P}_{>}(\{x, y, z\})$ as a subdomain.
} 
already observed that the domains $\mathcal{D}_{1}(x, y, z)-\mathcal{D}_{5}(x, y, z)$ are all weakly single-peaked. As in Section 2 above, the weak single-peakedness of $\mathcal{D}$ on all triples with respect to the same spectrum $>:=\bar{P}$ implies its weak single-peakedness globally with respect to $>$.

c) Finally, suppose that $\mathcal{D} \subseteq \mathcal{R}(X)$ is a semi-connected and even strongly minimally rich Condorcet domain. Then, for all triples $x, y, z \in X$ with $x \bar{P} y \bar{P} z$ the restriction $\mathcal{D}_{\{x, y, z\}}$ coincides with $\mathcal{D}_{1}(x, y, z)$ (all other semi-connected Condorcet domains on $\{x, y, z\}$ are not strongly minimally rich). By the arguments given in part a) this implies that $\mathcal{D} \subseteq \hat{\mathcal{R}}_{>}$. This completes the proof of Theorem 3.

While, for a given spectrum $>$, the Condorcet domain $\hat{\mathcal{R}}_{>}$is uniquely determined by the conditions in Theorem $3 \mathrm{c}$ ) and maximality, there are several non-isomorphic maximal single-peaked domains that satisfy the weaker richness property in part b). Figure 9 depicts four of them.
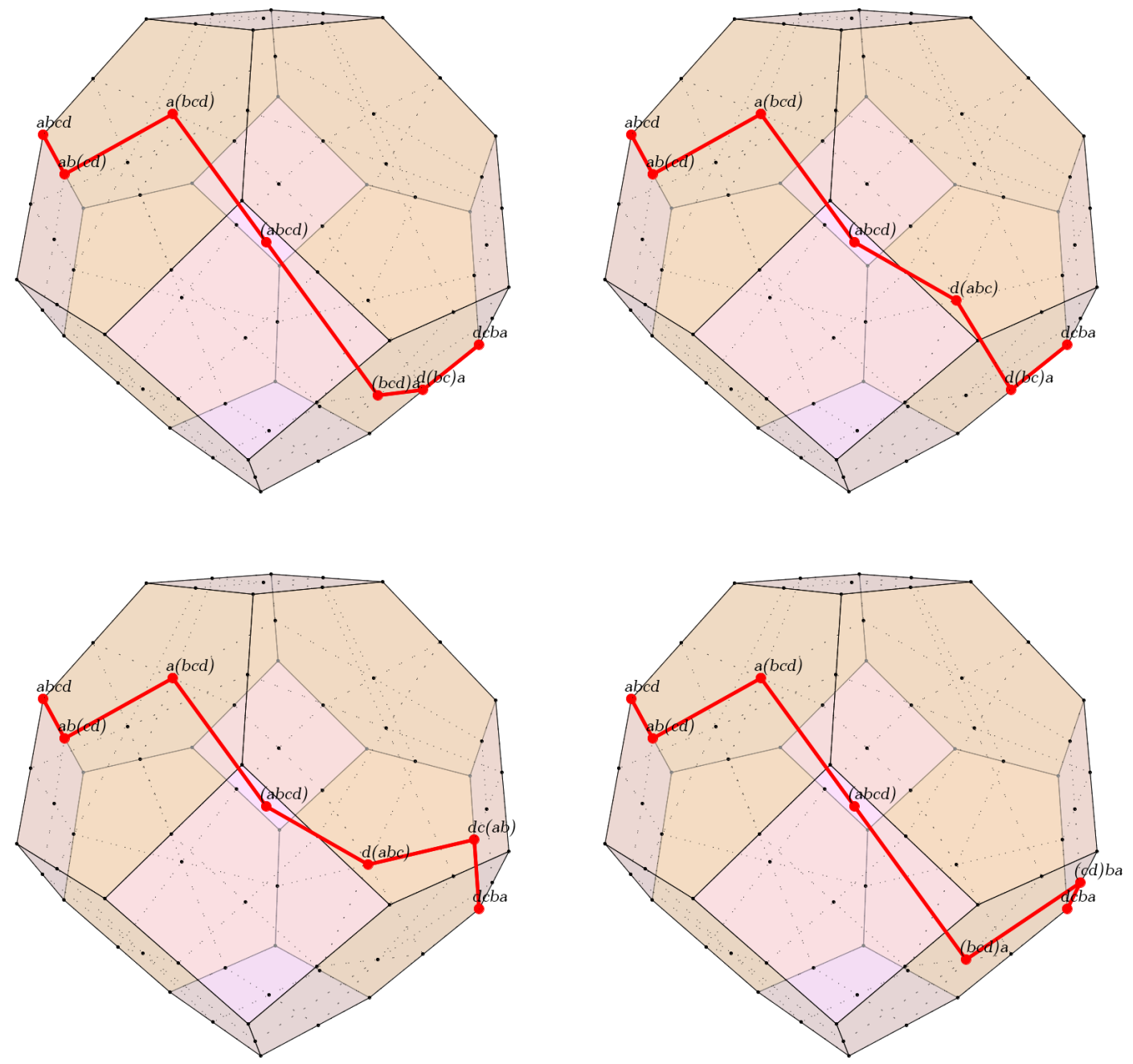

Fig. 9: Four maximal weakly single-peaked domains satisfying weak minimal richness. 
The reason for the multiplicity of weakly single-peaked Condorcet domains that satisfy the weak minimal richness condition is that the restrictions to different triples of alternatives may correspond to different types of Condorcet domains on the triples (for each triple $x, y, z \in X$, one of the domains $\left.\mathcal{D}_{1}(x, y, z)-\mathcal{D}_{5}(x, y, z)\right)$. By contrast, under the strong minimal richness conditions, the restrictions to all triples $x, y, z$ coincide uniformly with $\mathcal{D}_{1}(x, y, z)$.

\subsection{A dual characterization of the weakly single-dipped domain}

As in the case of linear orders, we obtain a corresponding characterization of the singledipped domain, as follows.

Theorem 4. a) For every linear order $>$ on $X$, there exists a unique maximal connected Condorcet domain $\check{\mathcal{R}}_{>} \subseteq \mathcal{R}(X)$ that contains the domain $\mathcal{S} \mathcal{D}_{>}$of all singledipped linear orders with respect to $>$. The domain $\check{\mathcal{R}}_{>}$is weakly single-dipped and connected. Evidently, $\check{\mathcal{R}}_{>}$has maximal width and contains, for every alternative in $X$, at least one order that has this alternative uniquely at the bottom.

b) Conversely, let $\mathcal{D} \subseteq \mathcal{R}(X)$ be a semi-connected Condorcet domain such that every alternative is among the worst alternatives for some order in $\mathcal{D}$. Then, $\mathcal{D}$ is weakly single-dipped.

c) Moreover, if $\mathcal{D} \subseteq \mathcal{R}(X)$ is a semi-connected Condorcet domain such that every alternative is uniquely worst for some order in $\mathcal{D}$, then $\mathcal{D} \subseteq \check{\mathcal{R}}_{>}$for some linear order $>$ on $X$. (In particular, $\mathcal{D}$ is weakly single-dipped.)

The proof of Theorem 4 is completely symmetric to the proof of Theorem 3 above; the details are left to the reader.

\subsection{A corollary for weakly single-crossing domains}

The following is a natural generalization of the single-crossing property for domains of weak orders. Say that a domain $\mathcal{D} \subseteq \mathcal{R}(X)$ is a weakly single-crossing domain if it can be written in the form $\mathcal{D}=\left\{R_{1}, \ldots, R_{m}\right\}$ such that, for all ordered pairs $x, y \in X$, the sets $\{R \in \mathcal{D}: x R y\}$ and $\{R \in \mathcal{D}: x \neg R y\}$ are connected in $\{1, \ldots, m\}$. As is easily verified, this condition implies that in the order $1, \ldots, m$, for all distinct alternatives $x, y \in X$, either (i) all weak orders $R_{j}$ that rank $x$ strictly above $y$ are followed by all weak orders $R_{j}$ that declare $x$ and $y$ as indifferent, and these are followed by all weak orders $R_{j}$ that rank $y$ strictly above $x$ or, conversely, (ii) all weak orders $R_{j}$ that rank $y$ strictly above $x$ are followed by all weak orders $R_{j}$ that declare $x$ and $y$ as indifferent, and these are followed by all weak orders $R_{j}$ that rank $x$ strictly above $y$ (cf. Rothstein $[1990,1991]$ ). 
Corollary 4. Let $\mathcal{D} \subseteq \mathcal{P}(X)$ be a weakly single-crossing domain.

a) If $\mathcal{D}$ is weakly minimally rich, then it is weakly single-peaked.

b) If every alternative in $X$ is among the worst alternatives in at least one order in $\mathcal{D}$, then $\mathcal{D}$ is weakly single-dipped.

c) If $\mathcal{D}$ is strongly minimally rich, then $\mathcal{D} \subseteq \hat{\mathcal{R}}_{>}$for some linear order $>$on $X$. (In particular, $\mathcal{D}$ is weakly single-peaked.)

d) If every alternative in $X$ is the uniquely worst alternative in at least one order in $\mathcal{D}$, then $\mathcal{D} \subseteq \check{\mathcal{R}}_{>}$for some linear order $>$on $X$. (In particular, $\mathcal{D}$ is weakly single-dipped.)

Proof. The proof follows from Theorems 3 and 4 since every weakly single-crossing domain $\mathcal{D}$ can be extended to a semi-connected weakly single-crossing domain $\mathcal{D}^{*} \supseteq \mathcal{D}$ as in the proof of Corollary 3. Here is a sketch how to construct the semi-connected domain $\mathcal{D}^{*} \subseteq \mathcal{R}(X)$. Given the weakly single-crossing domain $\mathcal{D}=\left\{R_{1}, \ldots, R_{m}\right\}$, let $R_{0}$ be any linear order satisfying, for all $x, y \in X$, (i) $x P_{m} y \Rightarrow y P_{0} x$, and (ii) $\left(x I_{m} y \& x P_{1} y\right) \Rightarrow x P_{0} y$, where $P_{m}, P_{1}, P_{0}$ are the asymmetric parts of $R_{m}, R_{1}, R_{0}$, respectively, and $I_{m}$ is the symmetric part of $R_{m}$; moreover, let $R_{m+1}:=R_{0}^{\text {inv }}$ be the inverse of $R_{0}$. Then consider the domain $\left\{R_{0}, R_{1}, \ldots, R_{m}, R_{m+1}\right\}$, where we allow for the possibility that $R_{0}=R_{1}$ and/or $R_{m}=R_{m+1}$. As is easily seen, the domain $\left\{R_{0}, R_{1}, \ldots, R_{m}, R_{m+1}\right\}$ is again weakly singlecrossing. Now fill all possible 'gaps' in an appropriate way, as in the proof of Corollary 3 above, whenever the interval $\left[R_{j}, R_{j+1}\right]$ contains weak orders distinct from both $R_{j}$ and $R_{j+1}$ for $j=0, \ldots, m$.

\section{Conclusion}

How restrictive is the assumption of single-peakedness as a domain restriction? In this paper it is argued that single-peakedness follows very naturally from transitivity of the majority relation for all odd profiles under a few simple and reasonable conditions of richness and connectedness. But transitivity of the majority relation for every odd profile is clearly a very demanding condition. As is well-known, its full strength is not needed in order to derive possibility results in social choice theory. For instance, the existence of non-dictatorial Arrovian aggregators and/or strategy-proof social choice functions can be demonstrated under much weaker domain restrictions (Kalai and Muller [1977]). Also in this context, richness and/or connectedness assumptions have frequently been imposed, and variants of the single-peakedness condition have been found to play an important role in the derivation of possibility results (Nehring and Puppe [2007], Chatterji et al. [2013], Chatterji and Massó [2015]). In a recent paper, Chatterji et al. [2016] have characterized a weaker notion of single-peakedness ('single-peakedness with respect to a tree') using strategy-proofness and other conditions imposed on random social choice functions. It 
seems a worthwhile task for future research to further explore whether, and how, the present methodology can contribute to our understanding of the weaker domain restrictions that still enable consistent preference aggregation and/or non-dictatorial strategyproof social choice. One important lesson that can be drawn from the present analysis in this context is that each of the conditions of (semi-)connectedness, minimal richness, and the presence of two completely reversed orders substantially restrict the combinatorial space of possibilities. Even if these conditions are justifiable by the specific context or application at hand, they cannot be considered mere 'technical' requirements nor innocuous assumptions. 


\section{Appendix: Maximal semi-connected Condorcet domains are (directly) connected}

In this appendix it is proved that all closed (in particular, all maximal) semi-connected Condorcet domains are in fact connected, and even satisfy a stronger property called direct connectedness. This holds both for domains of linear orders and domains of weak orders. These results and their proofs have been deferred to this appendix because they require some additional definitions and concepts; moreover, they are not needed to derive the results of Sections 2 and 3 above. $^{21}$ On the other hand, Propositions A.1 and A.2 below are included here because they describe a fundamental and remarkable property of Condorcet domains that turns out to be very useful in the classification of all maximal Condorcet domains (for small numbers of alternatives), see Dittrich [2016].

\section{A.1 The case of linear orders}

The domain $\mathcal{P}(X)$ of all linear orders on $X$ is naturally endowed with a betweenness structure as follows. An order $Q$ is between the orders $P$ and $P^{\prime}$ if $Q \supseteq\left(P \cap P^{\prime}\right)$, i.e., if $Q$ agrees with all binary comparisons in which $P$ and $P^{\prime}$ agree (Kemeny and Snell [1960]). ${ }^{22}$ The set of all orders that are between $P$ and $P^{\prime}$ is called the interval spanned by $P$ and $P^{\prime}$ and is denoted by $\left[P, P^{\prime}\right]$. The domain $\mathcal{P}(X)$ endowed with this betweenness relation is sometimes referred to as the permutahedron. A subset $\mathcal{D} \subseteq \mathcal{P}(X)$ is called median stable if, for any triple of elements $P_{1}, P_{2}, P_{3} \in \mathcal{D}$, there exists an element $P^{\text {med }}=P^{\text {med }}\left(P_{1}, P_{2}, P_{3}\right) \in \mathcal{D}$, the median order corresponding to $P_{1}, P_{2}, P_{3}$, such that

$$
P^{\text {med }} \in\left[P_{1}, P_{2}\right] \cap\left[P_{1}, P_{3}\right] \cap\left[P_{2}, P_{3}\right] .
$$

It is well-known that the median order of any triple $P_{1}, P_{2}, P_{3} \in \mathcal{P}(X)$, if it exists, is unique. The following observation is well-known but fundamental, and will be useful in the following (cf. [Nehring and Puppe, 2007, Cor. 5]).

Fact A.1. A triple $P_{1}, P_{2}, P_{3} \in \mathcal{P}(X)$ admits a median order if and only if the majority relation $P_{\pi}^{\text {maj }}$ corresponding to the profile $\pi=\left(P_{1}, P_{2}, P_{3}\right)$ is transitive, in which case $P^{\text {med }}\left(P_{1}, P_{2}, P_{3}\right)$ and $P_{\pi}^{\text {maj }}$ coincide. In particular, a domain $\mathcal{D}$ is a closed Condorcet domain if and only if it is median stable.

Recall that every maximal Condorcet domain $\mathcal{D}$ is closed in the sense that the majority relation of every odd profile from $\mathcal{D}$ is again an element of $\mathcal{D}$ (and not only of $\mathcal{P}(X)$ ). In particular, the maximal Condorcet domains are exactly the maximal median stable subsets of $\mathcal{P}(X)$.

A domain $\mathcal{D} \subseteq \mathcal{P}(X)$ is called directly connected if every pair $P_{1}, P_{m} \in \mathcal{D}$ is connected by a path $\left\{P_{1}, \ldots, P_{m}\right\} \subseteq \mathcal{D}$ such that for all $1 \leq j<k \leq m,\left\{P_{j}, P_{j+1}, \ldots, P_{k}\right\} \subseteq\left[P_{j}, P_{k}\right]$.

\footnotetext{
${ }^{21}$ Theorems 3a) and 4a) invoke Proposition A.2 in order to demonstrate the connectedness of $\hat{\mathcal{R}}_{>}$and $\check{\mathcal{R}}_{>}$, respectively; the connectedness of these two domains can also be directly verified.

${ }^{22}$ Some authors such as, e.g., Grandmont [1978] and Demange [2012] refer to orders that are between two others in this sense as 'intermediate' orders.
} 
A path as required in this definition is also called a direct path connecting $P_{1}$ and $P_{m}{ }^{23}$ Evidently, direct connectedness implies connectedness, but the converse does not hold. A simple example is the domain $\{x y z, y x z, y z x, z y x, z x y\} \subseteq \mathcal{P}(\{x, y, z\})$, which is connected but not directly connected (see Fig. 5 above). Indeed, the two orders $x y z$ and $z x y$ are connected, but not directly so. To see this, simply note that these two orders agree in their ranking of $x$ versus $y$, while all other orders on the unique path connecting them display the opposite ranking of $x$ versus $y$.

The following concepts will be useful. Say that two distinct orders $P, P^{\prime} \in \mathcal{P}(X)$ are $\mathcal{P}(X)$-neighbors if $\left[P, P^{\prime}\right]=\left\{P, P^{\prime}\right\}$. Note that $P$ and $P^{\prime}$ are $\mathcal{P}(X)$-neighbors if and only if they differ in the ranking of exactly one (adjacent) pair of alternatives. Moreover, for all subdomains $\mathcal{D} \subseteq \mathcal{P}(X)$, say that two distinct orders $P, P^{\prime} \in \mathcal{D}$ are $\mathcal{D}$-neighbors if $\left[P, P^{\prime}\right] \cap \mathcal{D}=\left\{P, P^{\prime}\right\}$. The following lemma provides a useful criterion for direct connectedness.

Lemma A.1. A domain $\mathcal{D} \subseteq \mathcal{P}(X)$ is directly connected if and only if all $\mathcal{D}$-neighbors are also $\mathcal{P}(X)$-neighbors.

Proof. Evidently, if $\mathcal{D}$ is directly connected, then all $\mathcal{D}$-neighbors must be $\mathcal{P}(X)$ neighbors. For the converse statement, we will use the following property which is known in the literature on abstract interval operators as the geometricity condition (cf. van de Vel [1993]) and which is easily verified in our case. For all $P, P^{\prime}, Q, Q^{\prime} \in \mathcal{P}(X)$,

$$
P, P^{\prime} \in\left[Q, Q^{\prime}\right] \text { and } P^{\prime} \in[Q, P] \Rightarrow P \in\left[P^{\prime}, Q^{\prime}\right] \text {. }
$$

Let $Q, Q^{\prime}$ be two distinct elements of $\mathcal{D}$, and suppose that all $\mathcal{D}$-neighbors are $\mathcal{P}(X)$ neighbors. We show by induction over $m:=\#\left[Q, Q^{\prime}\right]$ that there exists a direct path in $\mathcal{D}$ connecting $Q$ and $Q^{\prime}$. For $\#\left[Q, Q^{\prime}\right]=2$, i.e. $\left[Q, Q^{\prime}\right]=\left\{Q, Q^{\prime}\right\}$ there is nothing to show, since then $Q$ and $Q^{\prime}$ are $\mathcal{P}(X)$-neighbors. Thus, suppose $\#\left[Q, Q^{\prime}\right]>2$, i.e. suppose that $Q$ and $Q^{\prime}$ are not $\mathcal{P}(X)$-neighbors. By assumption, $Q$ and $Q^{\prime}$ cannot be $\mathcal{D}$-neighbors either, thus there exists $P \in\left[Q, Q^{\prime}\right] \cap \mathcal{D}$ distinct from both $Q$ and $Q^{\prime}$. Way may choose $P$ such that $P$ and $Q^{\prime}$ are $\mathcal{D}$-neighbors, hence by assumption also $\mathcal{P}(X)$-neighbors. By the induction hypothesis, there exists a direct path $\mathcal{A} \subseteq \mathcal{D}$ connecting $Q$ and $P$. Let $P^{\prime} \in \mathcal{A}$ be the neighbor of $P$ on this path. By the geometricity condition (A.1), we have $P \in\left[P^{\prime}, Q^{\prime}\right]$. From this, it follows immediately that $\mathcal{A} \cup\left\{Q^{\prime}\right\}$ is a direct path connecting $Q$ and $Q^{\prime}$, as desired.

Proposition A.1. Let $\mathcal{D} \subseteq \mathcal{P}(X)$ be semi-connected. If $\mathcal{D}$ is a closed Condorcet domain, then $\mathcal{D}$ is directly connected.

Proof. By Lemma A.1, it suffices to show that all $\mathcal{D}$-neighbors are $\mathcal{P}(X)$-neighbors. By contradiction, suppose that $P, P^{\prime} \in \mathcal{D}$ are distinct orders with $\left[P, P^{\prime}\right] \cap \mathcal{D}=\left\{P, P^{\prime}\right\}$ that differ in the ranking of more than one pair of alternatives, say $a P b$ and $c P d$ while $b P^{\prime} a$

\footnotetext{
${ }^{23}$ Sato [2013] uses the term 'connected without restoration' for what is called 'directly connected' here; for the relation to Sato's work see the remark at the end of Subsection A.2 below.
} 
and $d P^{\prime} c$ (note that it is not assumed that $\{a, b\} \cap\{c, d\}=\emptyset$ ). Observe that since $P$ and $P^{\prime}$ are $\mathcal{D}$-neighbors, one has $P^{\text {med }}\left(P, P^{\prime}, Q\right) \subseteq\left\{P, P^{\prime}\right\}$ for all $Q \in \mathcal{D}$.

Now, by the semi-connectedness, let $P_{1}, P_{m} \in \mathcal{D}$ be two completely reversed orders and let $\left\{P_{1}, \ldots, P_{m}\right\} \subseteq \mathcal{D}$ be a path connecting them. Without loss of generality, let $a P_{1} b$. Then, since $P_{m}$ is completely reversed, $b P_{m} a$. Thus, there exist (at least) two neighbors $P_{k}, P_{k+1} \in\left\{P_{1}, \ldots, P_{m}\right\}$ such that $a P_{k} b$ and $b P_{k+1} a$. There are two cases, either (i) $c P_{k} d$ and $c P_{k+1} d$, or (ii) $d P_{k} c$ and $d P_{k+1} c$. In case (i), the triple $P, P^{\prime}, P_{k+1} \in \mathcal{D}$ does not admit a median order (since neither $P \in\left[P^{\prime}, P_{k+1}\right]$, nor $P^{\prime} \in\left[P, P_{k+1}\right]$ ); in case (ii), the triple $P, P^{\prime}, P_{k} \in \mathcal{D}$ does not admit a median order (since neither $P \in\left[P^{\prime}, P_{k}\right]$, nor $\left.P^{\prime} \in\left[P, P_{k}\right]\right)$. Thus, in each case we obtain a contradiction to the assumption that $\mathcal{D}$ is a closed Condorcet domain using Fact A.1.

\section{A.2 The case of weak orders}

Recall that in the case of weak orders the appropriate notion of 'betweenness' is formalized by definition (3.1) (which coincides with the one given above in the case of linear orders). As in Section 3 above, two weak orders $R, R^{\prime}$ are $\mathcal{R}(X)$-neighbors if $\left[R, R^{\prime}\right]=\left\{R, R^{\prime}\right\},{ }^{24}$ and as in the previous subsection two weak orders $R, R^{\prime} \in \mathcal{D} \subseteq \mathcal{R}(X)$ are $\mathcal{D}$-neighbors if $\left[R, R^{\prime}\right] \cap \mathcal{D}=\left\{R, R^{\prime}\right\}$. The notion of direct connectedness is defined analogously to the case of linear orders, and by exactly the same argument as the one given in the proof of Lemma A.1 above, we obtain that a domain $\mathcal{D} \subseteq \mathcal{R}(X)$ is directly connected if and only if all $\mathcal{D}$-neighbors are also $\mathcal{R}(X)$-neighbors.

The following simple observation is useful (and straightforward to verify).

Fact A.2. Two distinct orders $R, R^{\prime}$ are neighbors if and only if either (i) $R \subseteq R^{\prime}$ and, for all $Q \in \mathcal{R}(X)$ with $R \subseteq Q \subseteq R^{\prime}$, we have $Q=R$ or $Q=R^{\prime}$, or (ii) $R^{\prime} \subseteq R$ and, for all $Q \in \mathcal{R}(X)$ with $R^{\prime} \subseteq Q \subseteq R$, we have $Q=R^{\prime}$ or $Q=R$.

Proposition A.2. Let $\mathcal{D} \subseteq \mathcal{R}(X)$ be semi-connected. If $\mathcal{D}$ is a closed Condorcet domain, then $\mathcal{D}$ is directly connected.

Proof. As in the proof of Proposition A.1 above, it suffices to show that all $\mathcal{D}$-neighbors are $\mathcal{R}(X)$-neighbors. By contradiction, suppose that $R, R^{\prime} \in \mathcal{D}$ are distinct orders with $\left[R, R^{\prime}\right] \cap \mathcal{D}=\left\{R, R^{\prime}\right\}$ that are not $\mathcal{R}(X)$-neighbors. Observe first that since $R$ and $R^{\prime}$ are $\mathcal{D}$-neighbors and since $\mathcal{D}$ is a closed Condorcet domain, one has $R^{\text {med }}\left(R, R^{\prime}, Q\right) \subseteq\left\{R, R^{\prime}\right\}$ for all $Q \in \mathcal{D}$.

Since $R, R^{\prime}$ are not $\mathcal{R}(X)$-neighbors, there exists $Q \in\left[R, R^{\prime}\right]$ but $Q \notin \mathcal{D}$, and we may assume without loss of generality that $Q$ is a $\mathcal{R}(X)$-neighbor of $R$. By Fact A.2, we have either (a) $R \subsetneq Q$ and, for all $Q^{\prime} \in \mathcal{R}(X)$ with $R \subseteq Q^{\prime} \subseteq Q, Q^{\prime}=R$ or $Q^{\prime}=Q$, or (b) $Q \subsetneq R$ and, for all $Q^{\prime} \in \mathcal{R}(X)$ with $Q \subseteq Q^{\prime} \subseteq R, Q^{\prime}=Q$ or $Q^{\prime}=R$.

Consider case (a) first. In this case, there exists $a, b \in X$ such that $b Q a$ but $b \neg R a$, hence by completeness $a R b$, and therefore also $a Q b$ and $b R^{\prime} a$. There are two subcases, either (i) $a R^{\prime} b$ or (ii) $a \neg R^{\prime} b$. Suppose first the latter, i.e. $a \neg R^{\prime} b$. By the semi-connectedness,

\footnotetext{
${ }^{24}$ In Section 3 above, we referred to $\mathcal{R}(X)$-neighbors simply as 'neighbors' since no confusion could arise; in the present context, we have to be more specific.
} 
let $R_{1}, R_{m} \in \mathcal{D}$ be two completely reversed (linear) orders and let $\left\{R_{1}, \ldots, R_{m}\right\} \subseteq \mathcal{D}$ be a path connecting them. Without loss of generality, let $a R_{1} b$ and $b \neg R_{1} a$. Then, since $R_{m}$ is completely reversed, we have $b R_{m} a$ and $a \neg R_{m} b$. Thus, there exist (at least) two $\mathcal{R}(X)$-neighbors $R_{k}, R_{k+1} \in\left\{R_{1}, \ldots, R_{m}\right\}$ such that $a R_{k} b, b \neg R_{k} a, a R_{k+1} b$ and $b R_{k+1} a$. But in this case, the triple $\left\{R, R^{\prime}, R_{k+1}\right\} \subseteq \mathcal{D}$ does not admit a median order, since neither $R \in\left[R^{\prime}, R_{k+1}\right]$ nor $R^{\prime} \in\left[R, R_{k+1}\right]$ (recall that the median order of any triple containing $R$ and $R^{\prime}$ must coincide either with $R$ or $R^{\prime}$ ). This shows that subcase (ii) is not possible. Thus, we must have $a R^{\prime} b$ as asserted in case (i), thus $a$ and $b$ are indifferent with respect to both orders $R^{\prime}$ and $Q$. Since $Q$ differs from $R^{\prime}$, there must exist at least one ordered pair $(c, d)$ of alternatives distinct from $(a, b)$ such that $R^{\prime}$ and $Q$ differ in their ranking of $c$ versus $d$. Since $R$ and $Q$ are $\mathcal{R}(X)$-neighbors, $R^{\prime}$ and $R$ must differ in their ranking of $c$ versus $d$ as well. Moreover, since $R_{k}$ and $R_{k+1}$ are $\mathcal{R}(X)$-neighbors which differ in the ranking of $a$ versus $b$, they must agree in their ranking of $c$ versus $d$. If this common ranking of $c$ versus $d$ coincides with that of $R$, then the triple $\left\{R, R^{\prime}, R_{k+1}\right\} \subseteq \mathcal{D}$ does not admit a median; on the other hand, if the common ranking of $R_{k}$ and $R_{k+1}$ vis-á-vis $(c, d)$ coincides with that of $R^{\prime}$, then the triple $\left\{R, R^{\prime}, R_{k}\right\} \subseteq \mathcal{D}$ does not admit a median order. In either case, we obtain a contradiction, which completes the proof of case (a).

The argument in case (b) is similar, but even simpler because there are no further subcases to consider. Indeed, suppose that $Q \in\left[R, R^{\prime}\right]$ is a $\mathcal{R}(X)$-neighbor of $R$ with $Q \subsetneq R$. Then, there exist $a$ and $b$ such that $b R a$ but $b \neg Q a$. By completeness of $Q$, we must have $a Q b$ and hence also $a R b$. Since $Q \in\left[R, R^{\prime}\right]$, we must also have $a R^{\prime} b$ and $b \neg R^{\prime} a$. Since $Q$ and $R^{\prime}$ are distinct they must differ in their ranking of some ordered pair $(c, d)$ distinct from $(a, b)$; and since $Q$ and $R$ are neighbors, they must coincide in their ranking vis-á-vis $(c, d)$. Let $R_{k}$ and $R_{k+1}$ be as in case (a); if their common ranking vis-á-vis $(c, d)$ coincides with that of $R$, the triple $\left\{R, R^{\prime}, R_{k}\right\} \subseteq \mathcal{D}$ does not admit a median order; on the other hand, if their common ranking vis-á-vis $(c, d)$ coincides with that of $R^{\prime}$, the triple $\left\{R, R^{\prime}, R_{k+1}\right\} \subseteq \mathcal{D}$ does not admit a median order. Thus, also in case (b) we obtain a contradiction to the assumption that $\mathcal{D}$ is a closed Condorcet domain. This completes the proof of Proposition A.2.

Remark. In a recent paper, Sato [2013] introduced the notion of connectedness without restoration for domains of linear orders that coincides with our notion of direct connectedness. He demonstrates the usefulness of this concept for describing domains on which strategy-proofness is equivalent to 'local' strategy-proofness. Among other things, he shows that the domain of all single-peaked linear preferences with respect to some fixed spectrum is connected without restoration. Proposition A.1 generalizes this to all semiconnected and closed Condorcet domains of linear orders, and Proposition A.2 further to the case of weak orders. The notions of semi-connectedness and direct connectedness as defined here readily generalize to corresponding conditions on subspaces of abstract aggregation spaces within the general judgement aggregation framework (cf. List and Puppe [2009]). The same arguments as employed here can be used to prove in general that any semi-connected median stable subspace of any aggregation space is directly connected. 


\section{References}

J.M. Abello. The weak Bruhat order of $S_{\Sigma}$, consistent sets, and Catalan numbers. SIAM Journal on Discrete Mathematics, 4(1):1-16, 1991.

K. J. Arrow. Social Choice and Individual Values. Wiley, New York, 1951.

N. Aswal, S. Chatterji, and A. Sen. Dictatorial domains. Economic Theory, 22:45-62, 2003.

D. Austen-Smith and J. Banks. Positive Political Theory I: Collective Preference. Michigan University Press, 1998.

M. Ballester and G. Haeringer. A characterization of the single-peaked domain. Social Choice and Welfare, 36:305-322, 2011.

D. Black. On the rationale of group decision-making. Journal of Political Economy, 56: 23-34, 1948.

C. Chameni-Nembua. Permutoèdre et choix social. Third cycle thesis. Universitè de Paris V, 1989.

S. Chatterji and J. Massó. On strategy-proofness and the salience of single-peakedness. mimeographed, 2015.

S. Chatterji and A. Sen. Tops-only domains. Economic Theory, 46:255-282, 2011.

S. Chatterji, R. Sanver, and A. Sen. On domains that admit well-behaved strategy-proof social choice functions. Journal of Economic Theory, 148:1050-1073, 2013.

S. Chatterji, A. Sen, and H. Zeng. A characterization of single-peaked preferences via random social choice functions. Theoretical Economics, 11:711-733, 2016.

V.I. Danilov and G.A. Koshevoy. Maximal condorcet domains. Order, 30(1):181-194, 2013.

V.I. Danilov, A.V. Karzanov, and G.A. Koshevoy. Condorcet domains of tiling type. Discrete Appl. Math., 160(7-8):933-940, 2012.

G. Demange. Majority relation and median representative ordering. SERIEs, 3(1-2): 95-109, 2012.

P. DeMarzo, D. Vayanos, and J. Zwiebel. Persuasion bias, social influence and unidimensional opinions. Quarterly Journal of Economics, 118:909-968, 2003.

T. Dittrich. All Condorcet domains on $n \leq 4$ alternatives. mimeographed, 2016.

J. Duggan. Preference exclusions for social rationality. Social Choice and Welfare, 46(1): 93-118, 2016. 
P. J. Egan. 'Do something' politics and double-peaked policy preferences. Journal of Politics, 76:333-349, 2014.

E. Elkind, P. Faliszewski, and P. Skowron. Characterizing the single-peaked single-crossing domain. In Proceedings of the Twenty-Eighth AAAI Conference on Artificial Intelligence, pages 654-660, 2014.

P. C. Fishburn. Acyclic sets of linear orders: A progress report. Social Choice and Welfare, 19:431-447, 2002.

P.C. Fishburn. Acyclic sets of linear orders. Social Choice and Welfare, 14:113-124, 1997.

W. Gaertner. Domain Conditions in Social Choice Theory. Cambridge Univ. Press, 2001.

A. Galambos and V. Reiner. Acyclic sets of linear orders via the bruhat orders. Social Choice and Welfare, 30(2):245-264, 2008.

J.S. Gans and M. Smart. Majority voting with single-crossing preferences. Journal of Public Economics, 59:219-237, 1996.

J.-M. Grandmont. Intermediate preferences and the majority rule. Econometrica, 46: 317-330, 1978.

K. Inada. A note on the simple majority decision rule. Econometrica, 32:525-531, 1964.

E. Kalai and E. Muller. Characterization of domains admitting nondictatorial social welfare functions and nonmanipulable voting procedures. Journal of Economic Theory, 16:457-469, 1977.

J. Kemeny and L. Snell. Mathematical Models in the Social Sciences. Ginn, 1960.

B. Klaus, H. Peters, and T. Storcken. Strategy-proof division of a private good when preferences are single-dipped. Economics Letters, 55:339-346, 1997.

C. List and C. Puppe. Judgement aggregation: A survey. In Paul Anand, Prasanta K. Pattanaik, and Clemens Puppe, editors, The Handbook of Rational and Social Choice, pages 457-482. Oxford University Press, 2009.

C. List, R. Luskin, J. Fishkin, and I. McLean. Deliberation, single-peakedness, and the possibility of meaningful democracy: Evidence from deliberative polls. The Journal of Politics, 75:80-95, 2013.

B. Monjardet. Condorcet domains and distributive lattices, 2007. Lecture Notes, Ghent (personal communication).

B. Monjardet. Acyclic domains of linear orders: A survey. In Steven J. Brams, William V. Gehrlein, and Fred S. Roberts, editors, The Mathematics of Preference, Choice and Order, Studies in Choice and Welfare, pages 139-160. Springer Berlin Heidelberg, 2009. 
H. Moulin. On strategy-proofness and single-peakedness. Public Choice, 35:437-455, 1980.

H. Moulin. Generalized condorcet winners for single-peaked and single-plateau preferences. Social Choice and Welfare, 1:127-147, 1984.

H. Moulin. Axioms of cooperative decision making. Cambridge University Press, 1988.

K. Nehring and C. Puppe. The structure of strategy-proof social choice I: General characterization and possibility results on median spaces. Journal of Economic Theory, 135: 269-305, 2007.

K. Nehring, M. Pivato, and C. Puppe. The Condorcet set: Majority voting over interconnected propositions. Journal of Economic Theory, 151:286-303, 2014.

K. Nehring, M. Pivato, and C. Puppe. Unanimity overruled: Majority voting and the burden of history. Journal of Theoretical Politics, forthcoming.

S. Obraztsova, E. Elkind, P. Faliszewski, and A. Slinko. On swap-distance geometry of voting. In $A A M A S$ '13 Proceedings of the international conference on autonomous agents and multi-agant systems, pages 383-390, 2013.

C. Puppe and A. Slinko. Condorcet domains, median graphs and the single-crossing property. mimeographed, 2015.

K. Roberts. Voting over income tax schedules. J. Public Economics, 8:329-340, 1977.

P. Rothstein. Order restricted preferences and majority rule. Social Choice and Welfare, 7(4):331-342, 1990.

P. Rothstein. Representative voter theorems. Public Choice, 72(2-3):193-212, 1991.

A. Saporiti. Strategy-proofness and single-crossing. Theoretical Economics, 4:127-163, 2009.

S. Sato. A sufficient condition for the equivalence of strategy-proofness and nonmanipulability by preferences adjacent to the sincere one. Journal of Economic Theory, 148: 259-278, 2013.

A. K. Sen. A possibility theorem on majority decisions. Econometrica, 34:491-499, 1966.

D. Spector. Rational debate and one-dimensional conflict. Quarterly Journal of Economics, 115:181-200, 2000.

M. van de Vel. Theory of Convex Structures. North-Holland, Amsterdam, 1993.

B. Ward. Majority voting and alternative forms of public enterprises. In J. Margolis, editor, The Public Economy of Urban Communities, pages 112-126, Baltimore, MD, 1965. Johns Hopkins Press. 


\section{Working Paper Series in Economics}

recent issues

No. 97 Clemens Puppe: The single-peaked domain revisited: A simple global characterization, November 2016

No. 96 David Burka, Clemens Puppe, Laszlo Szepesvary and Attila Tasnadi: Neural networks would 'vote' according to Borda's rule, November 2016

No. 95 Vladimir Korzinov and Ivan Savin: Pervasive enough? General purpose technologies as an emergent property, November 2016

No. 94 Francesco D'Acunto, Daniel Hoang and Michael Weber: The effect of unconventional fiscal policy on consumption expenditure, October 2016

No. 93 Andranik S. Tangian: The third vote experiment: VAA-based election to enhance policy representation of the KIT student parliament, September 2016

No. 92 Clemens Puppe and Arkadii Slinko: Condorcet domains, median graphs and the single-crossing property, June 2016

No. 91 Markus Höchstötter, Mher Safarian, Anna Krumetsadik: Analysis of stochastic technical trading algorithms, June 2016

No. 90 Nikolaus Schweizer and Nora Szech: Optimal revelation of life-changing information, May 2016

No. 89 Helena Barnard, Robin Cowan, Alan Kirman and Moritz Müller: Including excluded groups: The slow racial transformation of the South African university system, May 2016

No. 88 Aniol Llorente-Saguer, Roman M. Sheremeta and Nora Szech: Designing contests between heterogeneous contestants: An experimental study of tie-breaks and bid-caps in all-pay auctions, May 2016

No. 87 Johannes Karl Herrmann and Ivan Savin: Optimal policy Identification: Insights from the German electricity market, March 2016 